\title{
3-Hydroxymorphinan, a metabolite of dextromethorphan, protects nigrostriatal pathway against MPTP-elicited damage both in vivo and in vitro
}

\author{
Wei Zhang, ${ }^{\dagger} \dagger$ Eun-Joo Shin, ${ }^{\ddagger}$ Tongguang Wang,* Phil Ho Lee, ${ }^{\ddagger} \S$ Hao Pang,* \\ Myung-Bok Wie, ${ }^{ \pm,} \|$Won-Ki Kim, ${ }^{\ddagger, \pi}$ Seong-Jin Kim, ${ }^{\dagger+}$ Wen-Hsin Huang, ${ }^{+\ddagger}$ Yongjun Wang, ${ }^{\dagger}$ \\ Wanqin Zhang, $\$ \$$ Jau-Shyong Hong,* and Hyoung-Chun Kim ${ }^{\ddagger}, 1$ \\ *Neuropharmacology Section, Laboratory of Pharmacology and Chemistry, National Institute of \\ Environmental Health Science/National Institutes of Health, Research Triangle Park, North \\ Carolina, USA; ${ }^{\dagger}$ Department of Neurology, Beijing Tiantan Hospital, Capital University of Medical \\ Sciences, Beijing, China; ${ }^{\ddagger}$ Neuropsychopharmacology and Toxicology Program, College of Pharmacy, \\ Kangwon National University, Chunchon, South Korea; ${ }^{\circledR}$ Department of Chemistry, Kangwon \\ National University, Chunchon, South Korea; "Department of Veterinary Medicine, Kangwon \\ National University, Chunchon, South Korea; ${ }^{\mathbb{T}}$ Division of NanoSciences, Ewha Woman's University, \\ Seoul, South Korea; ${ }^{\dagger+}$ Laboratory of Cell Regulation and Carcinogenesis, NCI/NIH, Bethesda, \\ Maryland, USA; ${ }^{+\star}$ School of Pharmacy, National Defense Medical Center, National Defense \\ University, Taiwan; and ${ }^{\S}$ Department of Physiology, Dalian Medical University, Dalian, China
}

ABSTRACT We investigated the neuroprotective property of analogs of dextromethorphan (DM) in lipopolysaccharide (LPS) and 1-methyl-4-phenyl-1,2,3,6-tetrahydropyridine (MPTP) models to identify neuroprotective drugs for Parkinson's disease (PD). In vivo studies showed that daily injections with DM analogs protected dopamine (DA) neurons in substantia nigra pars compacta and restored DA levels in striatum using two different models for PD. Of the five analogs studied, 3-hydroxymorphinan (3-HM), a metabolite of DM, was the most potent, and restored DA neuronal loss and DA depletion up to $90 \%$ of the controls. Behavioral studies showed an excellent correlation between potency for preventing toxin-induced decrease in motor activities and neuroprotective effects among the DM analogs studied, of which 3-HM was the most potent in attenuating behavioral damage. In vitro studies revealed two glia-dependent mechanisms for the neuroprotection by 3-HM. First, astroglia mediated the 3-HM-induced neurotrophic effect by increasing the gene expression of neurotrophic factors, which was associated with the increased acetylation of histone H3. Second, microglia participated in 3-HM-mediated neuroprotection by reducing MPTP-elicited reactive microgliosis as evidenced by the decreased production of reactive oxygen species. In summary, we show the potent neuroprotection by 3-HM in LPS and MPTP PD models investigated. With its high efficacy and low toxicity, 3-HM may be a novel therapy for PD.-Zhang, W., Shin, E-J., Wang, T., Lee, P. H., Pang, H., Wie, M-B., Kim, W-K., Kim, S-J., Huang, W-H., Wang, Y., Zhang, W., Hong, J-S., Kim, H-C. 3-Hydroxymorphinan, a metabolite of dextromethorphan, protects nigrostriatal pathway against MPTP-elicited damage both in vivo and in vitro. FASEB J. 20, 2496-2511 (2006)
Key Words: PD - substantia nigra pars compacta (SNpc) $\cdot$ striatum $\cdot$ astroglia $\cdot$ neurotrophic factors $\cdot$ microglia $\cdot$ ROS

PARKINSON'S DisEASE (PD) IS A neurodegenerative disorder characterized by a chronic and progressive loss of dopamine (DA) neurons in substantia nigra pars compacta (SNpc), leading to movement disorders including dyskinesia, resting tremor, rigidity, and gait disturbance (1). Despite significant advances in understanding the pathological changes in PD, the etiology and the underlying mechanism responsible for the progressive nature of neurodegeneration remain poorly understood. Furthermore, current therapies are limited to relieving PD symptoms and are largely ineffective in halting the process of neurodegeneration.

We have reported that dextromethorphan (DM), a widely used anticough agent, protected DA neurons against inflammagen LPS in midbrain neuron-glia cultures (2) and neurotoxin 1-methyl-4-phenyl-1,2,3,6-tetrahydropyridine (MPTP)-elicited neurotoxicity in vivo (3) via an anti-inflammatory effect by preventing the over-activation of microglia. Furthermore, we recently reported that 3-hydroxymorphinan (3-HM), a metabolite of DM, exerted a more potent neuroprotection than DM against LPS-induced DA neurotoxicity in primary midbrain mixed neuron-glia cultures (4). We attribute the higher potency of 3-HM to its additional neurotrophic effect provided by astroglia

\footnotetext{
${ }^{1}$ Correspondence: Neuropsychopharmacology and Toxicology Program, College of Pharmacy, Kangwon National University, Chunchon 200-701, South Korea. E-mail: kimhc@kangwon.ac.kr doi: 10.1096/fj.06-6006com
} 
in addition to the anti-inflammatory activity shared by 3 -HM and DM (4).

To continue this research, we investigated the neuroprotective property of a variety of analogs of DM. In this paper, we report that of the five morphinan analogs studied, 3-HM provided the best neuroprotection against LPS- and MPTP-elicited DA neurotoxicity both in vivo and in vitro. Mechanistic studies showed that the neurotrophic effect was due to an increase in the production of several neurotrophic factors from astroglia, which may be associated with the increase in histone acetylation induced by 3-HM as revealed by the increased level of histone 3 protein. Additionally, 3-HM decreased MPTP-induced production of both the extracellular superoxide and intracellular reactive oxygen species (iROS), which may be the basis for the antiinflammatory mechanism of 3-HM.

\section{MATERIALS AND METHODS}

\section{Reagent}

3-HM was synthesized as described by Kim et al. (5). All materials related to cell cultures were obtained from Invitrogen (Carlsbad, CA, USA). Reagent from Sigma-Aldrich (St. Louis, MO, USA) included MPTP, $\mathrm{MPP}^{+}$, LPS, cytosine $\beta$-D-arabinofuranoside (Ara-c), leu-leu methyl ester (LME), superoxide dismutase (SOD), 3,3'-diaminobenzidine, and pentobarbital. Sources for other compounds included: chloral hydrate (Fluka, Buchs, SG, Switzerland), $\left[{ }^{3} \mathrm{H}\right] \mathrm{DA}(30$ $\mathrm{Ci} / \mathrm{mmol}$ ) (Perkin Elmer Life Sciences, Boston, MA, USA), polyclonal anti-TH antibody (Ab) (a gift from Dr. John Reinhard of GlaxoSmithKline, Research Triangle Park, NC, USA), Vectastain avidin-biotin complex (ABC) kit and biotinylated secondary Ab (Vector Laboratories, Burlingame, CA, USA), fluorescence probe 2',7'-dichlorodi-hydrofluorescein (DCFH-DA) (Molecular Probes, Eugene, OR, USA; Calbiochem, San Diego, CA, USA), WST-1 (Dojindo Laboratories, Gaithersburg, MD, USA), Trizol reagent (Invitrogen); RNeasy Kit (Qiagen, Valencia, CA, USA), SYBR green polymerase chain reaction (PCR) master mix (Applied Biosystems, Cheshire, UK), anti-acetyl-histone H3 rabbit polyclonal IgG (Upstate Biotechnology, Charlottesville, VA, USA), anti-histone H3 rabbit polyclonal IgG (Cell Signaling Technology, Beverly, MA, USA), $0.1 \%$ SDS minigels (Bio-Rad Laboratories, Hercules, CA, USA), PVDF membrane (Millipore, Bedford, MA, USA), and enhanced chemiluminescence (ECL) kit (Amersham Pharmacia Biotech., Piscataway, NJ, USA).

\section{Animal treatment}

All animals were treated in strict accordance with the NIH Guide for the Humane Care and Use of Laboratory Animals (NIH Guide for the Care and Use of Laboratory Animals). Eight-wk-old male C57BL/6J mice (Bio Genomics Inc., Charles River Technology, Gapyung-Gun, Gyeonggi-Do, South Korea) weighing $\sim 25 \pm 3 \mathrm{~g}$ were maintained on a 12:12 h light:dark cycle and fed ad libitum. They were adapted for 2 wk to the conditions described above before experimentation. To examine the effect of morphinans on MPTPinduced neurotoxicity, mice received daily MPTP injection [20 mg/ kg of MPTP.HCl (16.52 mg/ $\mathrm{kg}$ as a base), s.c.] for 7 consecutive days. Each morphinan (12 or $24 \mathrm{mg} / \mathrm{kg}$, i.p.) was administered $30 \mathrm{~min}$ before every MPTP injection for the last
3 days. Animals were sacrificed $24 \mathrm{~h}$ after the last MPTP injection.

For intrastriatal LPS injection, mice were deeply anesthetized using chloral hydrate $(300 \mathrm{mg} / \mathrm{kg}$, s.c.), then fixed on the stereotaxic frame (David Kopf Instruments, Tujunga, CA, USA). LPS [(L-7011, Sigma-Aldrich Co, St. Louis. MO, USA) $2 \mu \mathrm{g}$ in a volume of $2 \mu \mathrm{l}$ of PBS] was injected bilaterally to 0.7 $\mathrm{mm}$ anterior from bregma, $1.0 \mathrm{~mm}$ lateral from midline, and $3.4 \mathrm{~mm}$ from dura. Each morphinan analog (see structures in Fig. 1) was administered (12 or $24 \mathrm{mg} / \mathrm{kg}$, i.p.) twice, $4 \mathrm{~h}$ and 30 min before LPS treatment. Mice were sacrificed 3 wk after LPS injection. Striatal tissues after treatment with MPTP or LPS were rapidly dissected, immediately frozen in liquid nitrogen, then stored at $-80^{\circ} \mathrm{C}$ until use. The rest of mice were perfused transcardially with $50 \mathrm{ml}$ of PBS, followed by $200 \mathrm{ml}$ of $4 \%$ paraformaldehyde in PBS under anesthesia with pentobarbital $(50 \mathrm{mg} / \mathrm{kg}$, i.p.). Perfused brains were postfixed in $4 \%$ paraformaldehyde for $4 \mathrm{~h}$, then immersed in $30 \%$ sucrose in PBS until sink.

\section{Locomotor activity}

To determine the psychotropic effect of morphinans, mice received a daily injection of each morphinan (20 or 40

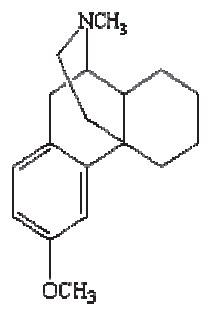

Dextromethorphan ( DM )<smiles>C=CCOc1ccc2c(c1)C13CCCCC1C(C2)N(C)CC3</smiles>

3-Allyloxy-17-methylmorphinan (3-AM)<smiles>Cc1ccc2c(c1)C13CCCCC1C(C2)N(C)CC3</smiles>

3-Methyl-N-methylmorphinan ( DF )

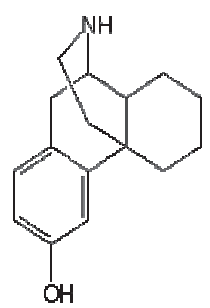

3-Hydroxymorphinan ( 3-HM)

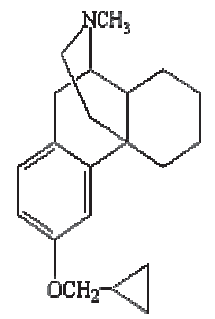

3-Cyclopropylmethoxy-17methylmorphinan (3-CM)<smiles>CC1Cc2ccc(O)cc2C2(CCCCC2C)C1</smiles>

Dextrorphan (DX)
Figure 1. Structure of morphinan compound DM and its analogs. Structure of morphinan compound DM and its analogs, including 3-hydroxymorphinan (3-HM), 3-allyloxy-17methylmorphinan (3-AM), 3-cyclopropylmethoxy-17-methylmorphinan (3-CM), 3-methyl-N-methyl-morphinan (DF), and dextrorphan (DX), are shown in Fig. 1. 
$\mathrm{mg} / \mathrm{kg}$, i.p.) for 7 days. Ten minutes after the last injection, locomotor activity was measured for $30 \mathrm{~min}$. After measuring locomotor activity (i.e., $40 \mathrm{~min}$ after the last injection), the "absolute turn angular" was analyzed in a 3 min monitoring period using an automated video tracking system (Noldus Information Technology, Wagenin, the Netherlands) to examine locomotor patterns (6). Eight test boxes $(40 \times 40 \times 30$ $\mathrm{cm}$ high) were operated simultaneously by an IBM computer. Animals were studied individually during locomotion in each test box, where they were adapted for 10 min before starting the experiment. The distance traveled in centimeters by the animals in horizontal locomotor activity was analyzed. Data were collected and analyzed between 0900 and $1700 \mathrm{~h}$.

\section{Conditioned place preference test}

Mice received an i.p. injection of saline just before entering the white or black compartment. Each compound (20 or 40 $\mathrm{mg} / \mathrm{kg}$, i.p.) dissolved in saline $(0.1 \mathrm{ml} / 10 \mathrm{~g})$ was administered immediately before the mice were placed in the white compartment. On day 1 , the mice were preexposed to the test apparatus for $15 \mathrm{~min}$. The guillotine style doors were raised and mice were allowed to move freely between the two compartments. On day 2, the time each mouse spent in each compartment was recorded for $15 \mathrm{~min}$. On days 3, 5, 7, 9, 11, and 13 , the mice were injected with each drug before being confined to the white compartment, the nonpreferred side, for $40 \mathrm{~min}$. On days 4, 6, 8, 10, and 12, the mice were injected with saline before being confined to the black compartment, the preferred side, for $40 \mathrm{~min}$. On day 14, the guillotine doors were raised. The mice were initially placed in the tunnel and the time spent by the mice in the two compartments was recorded for $15 \mathrm{~min}$. The scores were calculated from the differences in the time spent in the white compartment in the testing and pretesting phases $(6,7)$. Data were analyzed between 0900 and $1700 \mathrm{~h}$.

\section{Measurement of MPP+ in striatal extract after the last treatment with MPTP}

Striatal tissue samples were homogenized in buffer $[0.4 \mathrm{M}$ perchloric containing sodium metabisulphite $(0.1 \%, \mathrm{w} / \mathrm{v})$, EDTA $(0.01 \%, \mathrm{w} / \mathrm{v})$, and cysteine $(0.1 \%, \mathrm{w} / \mathrm{v})]$. An aliquot $(100 \mu \mathrm{l})$ of homogenizing buffer was used per milligram of tissue to produce a final tissue concentration of $0.01 \mathrm{~g} / \mathrm{ml}$. Samples were centrifuged for $10 \mathrm{~min}\left(10,000 \mathrm{rpm} ; 4^{\circ} \mathrm{C}\right)$ and the supernatant was retained for subsequent analysis of MPP + levels using LC-MS/MS. A stock solution of MPP+ was prepared in homogenization buffer at a concentration of 1.0 $\mathrm{mg} / \mathrm{ml}$. This solution was stored at $4^{\circ} \mathrm{C}$; all other solutions were prepared by diluting this stock solution with homogenization buffer. Buffer A was composed of $0.1 \%$ formic acid in $\mathrm{H}_{2} \mathrm{O}(\mathrm{v} / \mathrm{v})$. Buffer B consisted of $0.1 \%$ formic acid in acetonitrile $(\mathrm{v} / \mathrm{v})$. A linear gradient elution profile from $100 \%$ A to $100 \% \mathrm{~B}$ over $5 \mathrm{~min}$ at a flow rate of $0.3 \mathrm{ml} / \mathrm{min}$ was used for the separation, with a 3 min re-equilibration between injections.

The HPLC system was composed of two Jasco model PU-1585 HPLC pumps (Great Dunmow, UK), an HTS PAL autosampler fitted with a twin PAL stackcooler and twin six port injection valves with a $20 \mu$ l injection loop (CTC Analytics, Zwingen, Switzerland). The chromatography of $\mathrm{MPP}+$ was carried out using a $100 \mathrm{~mm} \times 2.1 \mathrm{~mm}$ i.d., $3 \mu \mathrm{m}$, Atlantis HILIC silica column (Waters, Milford, MA, USA), maintained at $40^{\circ} \mathrm{C}$. Eluates were detected using a Sciex API 4000 triquadrupole mass spectrometer equipped a Turboionspray source (TSQ Quantum Ultra, Thermo, USA). The ion spray voltage was set at $5 \mathrm{kV}$ and source temperature at $500^{\circ} \mathrm{C}$.
The mass spectrometer was operated in the positive ion eletrospray mode with the following parameters: declustering potential $60 \mathrm{~V}$, focusing potential $10 \mathrm{~V}$, entrance potential $-10 \mathrm{~V}$, collision cell exit potential $11 \mathrm{~V}$. Nitrogen was used for both the curtain and collision gas with an ion energy of $37 \mathrm{eV}$. $\mathrm{MPP}+$ was monitored using a molecular reaction monitoring mode with the transition $\mathrm{m} / \mathrm{z} 170.4 \rightarrow 127.9(8)$.

\section{Immunohistochemistry and cell counting in the SNpc of C57 mice}

The brains were cut on a horizontal sliding microtome into $35 \mu \mathrm{m}$ transverse free-floating sections. Four to 5 brain sections of $\mathrm{SNpc}$ were collected at intervals of $140 \mu \mathrm{m}$. Immunohistochemistry was performed as described previously (9). Briefly, prior to incubation with the primary antibodies, sections were preincubated with $0.3 \%$ hydrogen peroxide in PBS for $30 \mathrm{~min}$, then in PBS containing $0.4 \%$ Triton X-100 for $20 \mathrm{~min}$ and 1\% normal serum for $20 \mathrm{~min}$. After $48 \mathrm{~h}$ incubation with the anti-TH $(1: 5000)$ at $4^{\circ} \mathrm{C}$, sections were incubated with the biotinylated anti-mouse IgG $(1: 1000)$ for $1 \mathrm{~h}$ and immersed in Vectastain $\mathrm{ABC}$ reagents for 1 h. 3,3'-Diaminobenzidine was used as a chromogen. The bound complex was visualized using 3,3'-diaminobenzidine. Digital images of $\mathrm{TH}$ immunoreactive (TH-ir) neurons in $\mathrm{SNpc}$ were acquired at $40 \times$ magnifications on an Olympus microscope (Olympus ${ }^{\circledR}$, Tokyo, Japan) using an attached Polaroid digital microscope camera (Polaroid ${ }^{\circledR}$, Cambridge, MA, USA) and IBM PC. A region of interest (ROI) was created by outlining $\mathrm{SNpc}$ using Optimas ${ }^{\circledR}$ version 6.51 (Media Cybernetics, Inc., Silver Spring, MD, USA). Cell counting was performed blindly by two investigators and results were obtained from the average. A mean value for the number of SNpc TH-ir neurons was then deduced by averaging the counts of $4 \sim 5$ sections for each animal. Results were indicated as $\%$ of saline (+saline)-treated group.

\section{Neurochemical analysis of the contents of striatal neurotransmitters}

The levels of DA and its metabolites 3,4-dihydroxyphenylacetic acid (DOPAC) and homovanillic acid (HVA) were determined by HPLC coupled with electrochemical detection as described $(9,10)$. Briefly, striatal tissues were sonicated in $0.2 \mathrm{M}$ perchloric acid (PCA) $(20 \% \mathrm{~W} / \mathrm{V})$ containing the internal standard 3,4-dihydroxybenzylamine (10 $\mathrm{mg}$ wet tissue $/ \mathrm{ml}$ ). The homogenate was centrifuged and a $20 \mu \mathrm{l}$ aliquot of the supernatant was injected into the HPLC equipped with a $3 \mu \mathrm{m} \mathrm{C}_{18}$ column. The mobile phase was comprised of $26 \mathrm{ml}$ of acetonitrile, $21 \mathrm{ml}$ of tetrahydrofuran, and $960 \mathrm{ml}$ of $0.15 \mathrm{M}$ monochloroacetic acid $(\mathrm{pH} 3.0)$ containing $50 \mathrm{mg} / \mathrm{L}$ of EDTA and $200 \mathrm{mg} / \mathrm{L}$ of sodium octyl sulfate. The amount of DA, DOPAC, and HVA were determined by comparison of peak height ratio of tissue sample with standards and expressed in nanograms per gram of wet weight of tissue.

\section{Preparation of cell cultures}

Fisher 344 rat and C57BL/6J mice were purchased from Jackson Laboratories (Bar Harbor, ME, USA). Breeding schedules for the rat and mice were designed to achieve accurate timed-pregnancy of $14 \pm 0.5$ and $13 \pm 0.5$ days, respectively. Animals were maintained and bred in a strict pathogen free environment. Housing, breeding, and experimental use of the animals were performed in strict accordance with the National Institutes of Heath guidelines. Various primary cultures used in this study were described below. 
Primary midbrain neuron-glia cultures were prepared from the brains of embryonic day $14 \pm 0.5$ days of Fisher 344 rats and $13 \pm 0.5$ days of C57BL/6J mice as described previously (4). Briefly, the ventral midbrain tissues were removed and dissociated by a mild mechanical trituration. Rat (mice) cells were seeded at $5 \times 10^{5} /$ well $\left(1 \times 10^{5} /\right.$ well) in 24-well (96-well) culture plates precoated with poly-D-lysine $(20 \mu \mathrm{g} / \mathrm{ml})$ and maintained at $37^{\circ} \mathrm{C}$ in a humidified atmosphere of $5 \% \mathrm{CO}_{2}$ and $95 \%$ air in $0.5 \mathrm{ml}(200 \mu \mathrm{l})$ maintenance medium that consisted of minimum essential medium (MEM) containing $10 \%$ heat-inactivated FBS, $10 \%$ heat-inactivated horse serum (HS), $1 \mathrm{~g}$ /L glucose (Glc), $2 \mathrm{mM}$ L-glutamine, $1 \mathrm{mM}$ sodium pyruvate, $100 \mu \mathrm{M}$ nonessential amino acids, $50 \mathrm{U} / \mathrm{ml}$ penicillin, and $50 \mu \mathrm{g} / \mathrm{ml}$ streptomycin. Three days after the initial seeding, $0.5 \mathrm{ml}$ of fresh maintenance medium was added to each well of 24-well culture plates. Seven (2)-day-old 24 (96)-well cultures were used for treatment. The composition of the cultures at the time of treatment was $\sim 48 \%$ astroglia, $11 \%$ microglia, and $40 \%$ neurons in which $\sim 1 \%$ were $\mathrm{TH}$-ir neurons.

\section{Primary midbrain neuron-enriched cultures}

To obtain rat primary neuron-enriched cultures, $24 \mathrm{~h}$ after seeding the cells, Ara-c was added to a final concentration of $7.5 \mu \mathrm{M}$ to suppress glial proliferation (11). Three days later cultures were changed back to maintenance medium and were used for treatment 7 days after initial seeding. This method typically can enrich for neurons to $>95 \%$ purity.

\section{Primary microglia-enriched cultures}

Primary microglia-enriched cultures were prepared from the whole brains of 1-day-old Fisher 344 rat pups following our described protocol (12). Briefly, brain tissues were triturated after removing the meninges and blood vessels. Cells $\left(5 \times 10^{7}\right)$ were seeded in a $150 \mathrm{~cm}^{2}$ cultures flask. After a confluent monolayer of glia cells had been obtained, microglia were shaken off, collected (a purity of $>98 \%$ ), and seeded onto 96-well plates for extracellular superoxide and iROS assay.

\section{Primary midbrain astroglia-enriched cultures}

After removing loosely attached microglia by shaking three times, the remaining astroglia were detached with trypsinEDTA and seeded in the same culture medium as that used for microglia cultures. After three consecutive passages, a purity of $>98 \%$ of astroglia preparation can be achieved and used to measure 3-HM-induced gene expression of neurotrophic factors using reverse transcription (RT) and real-time quantitative PCR.

\section{Primary midbrain reconstituted neuron-microglia cultures}

To explore the role of microglia on 3-HM-exerted DA neuroprotection in the primary midbrain mixed neuron-glia culture system, the reconstituted cultures were established by adding $10 \%\left(5 \times 10^{4} /\right.$ well $)$ of microglia back to neuronenriched cultures as described previously (13). (In normal neuron-glia cultures, the composition of microglia is $\sim 10 \%$ of the total cells.)
Rat primary neuron-astroglia cocultures were obtained by suppressing microglial proliferation with $1.5 \mathrm{mM} \mathrm{LME} 24 \mathrm{~h}$ after seeding the cells, as described previously (13). Three days later cultures were changed back to maintenance medium and used for treatment 7 days after initial seeding. The composition of the cells at the time of treatment was $\sim 54 \%$ astrocytes, $1 \%$ microglia, and $45 \%$ neurons.

\section{DA uptake assay}

$\left[{ }^{3} \mathrm{H}\right] \mathrm{DA}$ uptake assay was performed as described previously (12). Cultures were incubated for $20 \mathrm{~min}$ at $37^{\circ} \mathrm{C}$ with $1 \mu \mathrm{M}$ $\left[{ }^{3} \mathrm{H}\right] \mathrm{DA}$ in Krebs-Ringer buffer (16 mU sodium phosphate, $119 \mathrm{mM} \mathrm{NaCl}, 4.7 \mathrm{mM} \mathrm{KCl}, 1.8 \mathrm{mM} \mathrm{CaCl}_{2}, 1.2 \mathrm{mM} \mathrm{MgSO}_{4}$, $1.3 \mathrm{mM}$ EDTA, and $5.6 \mathrm{mM} \mathrm{Glc}$; pH 7.4). After washing three times with ice-cold Krebs-Ringer buffer, cells were collected in $\mathrm{NaOH}(1 \mathrm{~N})$. Radioactivity was determined by liquid scintillation counting. Nonspecific DA uptake observed in the presence of mazindol $(10 \mu \mathrm{M})$ was subtracted.

\section{Immunocytochemical staining}

DA neurons were immunostained with anti-TH Ab. Briefly, $3.7 \%$ formaldehyde-fixed cultures were treated with $1 \%$ hydrogen peroxide ( $20 \mathrm{~min}$ ), followed by sequential incubation with blocking solution (30 min), primary $\mathrm{Ab}$ (overnight, $\left.4^{\circ} \mathrm{C}\right)$, biotinylated secondary $\mathrm{Ab}(2 \mathrm{~h})$, and $\mathrm{ABC}$ reagents $(40$ min). Color was developed with 3, 3'diaminobenzidine. For morphological analysis, images were recorded with an inverted microscope (Nikon, Tokyo, Japan) connected to a charged-coupled device camera (DAGE-MTI of MC, Inc., Michigan City, IN, USA) operated with the MetaMoph software (Universal Imaging Corporation, Downingtown, PA, USA). For visual counting DA neurons, nine representative areas per well of the 24-well plate were counted under the microscope at $100 \times$ magnification. To measure the average DA neuronal dendrite, 50 representative DA neurons in each well and three wells for each treatment condition were selected.

\section{Expressions of neurotrophic factors from astroglia using RT and real-time PCR}

Astroglia-enriched cultures treated with 3-HM (5 $\mu \mathrm{M})$ for 3, 6 , 12 , and $24 \mathrm{~h}$ were detached with trypsin-EDTA. Total RNA was isolated with Trizol reagent, followed by purification. Forward and reverse primers for selected neurotrophic factors were designed using ABI Primer Express software (Applied Biosystems, Foster City, CA, USA). The sequences of the primers were $\beta$-actin: TCCTCCTGAGCGCAAGTACTCT (F), GCTCAGTAACAGTCCGCCTAGAA (R); ADNF: TGCAGTCTGCTAATGCCTCTTC (F), ACTCTGGATGCCTGTGACTGAGA (R); GDNF: GAGAGAGGAACCGGCAAGCT (F), GTTAAGACGCACCCCCGATT (R); NT3: CACCACGGAGGAAACGCTAT (F), CACAGGCTCTCACTGTCACACA (R); b-FGF: GAACGCCTGGAGTCCAATAACT (F), GTTCGTTTCAGTGCCACATACC (R); epidermal growth factor (EGF): GCCACTGGGTCCGAAACA (F), TACATGCACACGCCACCATT (R); TGF- $\beta$ 1: GCCTGAGTGGCTG TCTTTTGA (F), GAAGCGAAAGCCCTGTATTCC (R). Insulin-like growth factor (IGF)-1: ACAGGCTATGGCTCCAGCATT (F), GATCACAGCTCCGGAAGCAA(R); TGF- $\alpha$ : CCCAGGTTGGCCTTGAAGT (F), TGGGTGCGGTGCTGTACA (R). RT and realtime PCR were utilized for analysis of the gene expression of neurotrophic factors using forward and reverse primers for the selected genes (14). Total RNA was reversely transcribed 
with MuLV reverse transcriptase and oligo-dT primers, and subjected to real-time PCR analysis using SYBR green PCR master mix (Applied Biosystems, Cheshire, UK). The relative differences in expression between groups were determined using cycle time $(\mathrm{Ct})$ values as follows: the $\mathrm{Ct}$ values for the genes of interest were first normalized with $\beta$-actin of the same sample, then the relative differences between control and treatment groups were calculated and expressed as relative increases, setting control as $100 \%$. Assuming that the Ct value is reflective of the initial starting copy and that there is $100 \%$ efficiency, a difference of one cycle is equivalent to a 2-fold difference in starting copy. Standard curve analysis was performed and used for the calculation.

\section{Western blot analysis of acetylated histone $\mathrm{H3}$ by 3-HM}

Astroglia-enriched cultures treated with 3-HM (5 $\mu \mathrm{M})$ for 1, 3, 6,12 , and $24 \mathrm{~h}$ were detached by scraping in sampling buffer containing $10 \mathrm{mM}$ HEPES ( $\mathrm{pH} 7.9$ ), $1.5 \mathrm{mM} \mathrm{MgCl}_{2}, 10 \mathrm{mM}$ $\mathrm{KCl}, 1.5 \mathrm{mM}$ phenylmethylsulfonyl fluoride, and $0.5 \mathrm{mM}$ dithiothreitol. Histones were isolated by means of acid extraction. Isolated histones were adjusted to equal protein concentrations and separated by $4-12 \%$ polyacrylamide- $0.1 \%$ SDS minigels, then transferred onto a PVDF membrane. Blots were incubated with primary $\mathrm{Ab}$ against acetylated histone $\mathrm{H} 3$, then probed with a horseradish peroxidase-conjugated secondary Ab. Detection was performed by using ECL via ECL kit.

\section{Assay of extracellular superoxide production in mouse} primary mixed midbrain neuron-glia cultures

The production of extracellular superoxide was determined by measuring the SOD-inhibited reduction of the tetrazolium salt WST-1, originally reported by Tan and Berridge (15) and by Peskin and Winterbourn (16) and adapted for neural cultures by Liu et al. $(12,17)$. To measure the reactive microgliosis-related release of superoxide, neuron-glia cultures in 96-well culture plates were pretreated for $30 \mathrm{~min}$ with vehicle alone or $0.5-1 \mu \mathrm{M} 3-\mathrm{HM}$ prior to treatment with $\operatorname{MPTP}(0.5 \mu \mathrm{M})$ or $\mathrm{MPP}^{+}(0.25 \mu \mathrm{M})$ in the $100 \mu$ l treatment medium/well (phenol red-free MEM, containing 2\% FBS and $2 \%$ HS) following the described protocol (3). Two and 5 days after MPTP or $\mathrm{MPP}^{+}$treatment, the desired concentrations of 3-HM were added back again to the corresponding 3-HMtreated cultures. On days 4 and $7,75 \mu$ l of WST-1 (final concentration: $1 \mathrm{mM}$ ) in treatment medium without and with SOD (final concentration: $50 \mathrm{U} / \mathrm{ml}$ ) was added to each well. Absorbance at $450 \mathrm{~nm}$ was read with a SpectraMax Plus microplate spectrophotometer (Molecular Devices, Palo Alto, CA, USA). The difference in absorbance observed in the absence and presence of SOD was considered to be the amount of superoxide produced. Results are expressed as a percentage of vehicle-treated control cultures.

\section{Determination of the levels of iROS in C57BL/6J mouse primary midbrain mixed neuron-glia cultures}

The level of iROS in C57BL/6J mouse-derived primary midbrain mixed neuron-glia cultures was measured using a fluorescent probe, DCFHDA, as described (18), with modifications (19). The method of treatment was the same as that for superoxide assay described above. Briefly, 4 and 7 days after treatment, cultures were treated with $10 \mu \mathrm{M}$ DCFHDA diluted in phenol red-free HBSS containing $2 \%$ FBS and $2 \%$ HS. After an additional $2 \mathrm{~h}$ of incubation at $37^{\circ} \mathrm{C}$, fluorescence intensity was measured at $485 \mathrm{~nm}$ for excitation and $530 \mathrm{~nm}$ for emission using a SpectraMax Gemini XS fluorescence microplate reader (Molecular Devices).

\section{Statistical analysis}

Statistical significance of the differences was determined by ANOVA, followed by Bonferroni's $t$ test using the StatView program (Abacus Concepts, Inc., Berkeley, CA, USA) except for Figs. 2, 3, 9 and Table 1 and Table 2, in which 1-way ANOVA with Fischer's PLSD test using Statcel ${ }^{\circledR}$ software (OMS, Saitama, Japan) was used. A value of $P<0.05$ was considered statistically significant.

\section{RESULTS}

\section{3-HM protected against DA neurodegeneration induced by MPTP and LPS in vivo}

Two in vivo rodent PD models were used to determine the neuroprotective effect of 3-HM and related morphi-

TABLE 1. 3-HM is the most potent morphinan in attenuation of the depletion of DA and its metabolites DOPAC and HVA in striatum induced by LPS in vivo ${ }^{a}$

\begin{tabular}{lccc}
\hline \hline & \multicolumn{3}{c}{ Concentration (ng/100 mg wet tissue) } \\
\cline { 2 - 4 } Compound & DA & DOPAC & HVA \\
\hline Saline + saline & $1041 \pm 106$ & $145 \pm 13$ & $118 \pm 11$ \\
Saline + LPS & $445 \pm 59^{\#}$ & $54 \pm 9^{\#}$ & $61 \pm 8^{\#}$ \\
DM (12 mg/kg) + LPS & $626 \pm 72$ & $65 \pm 8$ & $79 \pm 8$ \\
DM (24 mg/kg) + LPS & $889 \pm 74^{* * *}$ & $90 \pm 14^{* * *}$ & $93 \pm 13^{* *}$ \\
3-HM (12 mg/kg) + LPS & $689 \pm 73$ & $80 \pm 10$ & $80 \pm 8$ \\
3-HM (24 mg/kg) + LPS & $935 \pm 85^{* * *}$ & $106 \pm 11^{* * *}$ & $99 \pm 12^{* *}$ \\
3-AM (12 mg/kg) + LPS & $466 \pm 58$ & $64 \pm 8$ & $73 \pm 8$ \\
3-AM (24 mg/kg) + LPS & $485 \pm 67$ & $64 \pm 8$ & $75 \pm 10$ \\
3-CM (12 mg/kg) + LPS & $498 \pm 82$ & $69 \pm 8$ & $79 \pm 8$ \\
3-CM (24 mg/kg) + LPS & $687 \pm 53^{*}$ & $82 \pm 6 *$ & $87 \pm 5^{*}$ \\
DF (12 mg/kg) + LPS & $555 \pm 86$ & $56 \pm 9$ & $60 \pm 7$ \\
DF (24 mg/kg) + LPS & $552 \pm 77$ & $55 \pm 8$ & $63 \pm 8$ \\
\hline
\end{tabular}

${ }^{a}$ LPS $(2 \mu \mathrm{g}$ in a volume of $2 \mu \mathrm{l}$ of PBS) was injected into both sides of the striatum of C57BL/6J mice. Each morphinan was administered twice $(4 \mathrm{~h}$ and $30 \mathrm{~min}$ ) before intrastriatal injection with LPS. Mice were sacrificed $3 \mathrm{wk}$ after last LPS injection. Each value is the mean \pm se from 12 animals. ${ }^{\#} P<$ 0.01 vs. saline + saline; $* P<0.05, * * P<0.02$, and $* * * P<0.01$ vs. saline + LPS, respectively. 


\begin{tabular}{lccc}
\hline \hline & \multicolumn{3}{c}{ Concentration $(\mathrm{ng} / 100 \mathrm{mg}$ wet tissue) } \\
\cline { 2 - 4 } Compound & \multicolumn{1}{c}{ DA } & DOPAC & HVA \\
\hline Saline + saline & $1056 \pm 96$ & $146 \pm 15$ & $118 \pm 13$ \\
Saline + LPS & $403 \pm 60^{\#}$ & $49 \pm 8^{\#}$ & $59 \pm 11^{\#}$ \\
DM (12 mg/kg) + LPS & $498 \pm 69$ & $66 \pm 9$ & $80 \pm 8$ \\
DM (24 mg/kg) + LPS & $658 \pm 104^{* * *}$ & $82 \pm 12^{* *}$ & $92 \pm 11^{* *}$ \\
3-HM (12 mg/kg) + LPS & $571 \pm 82$ & $67 \pm 9$ & $81 \pm 8$ \\
3-HM (24 mg/kg) + LPS & $823 \pm 57^{* * *}$ & $95 \pm 10^{* * *}$ & $101 \pm 10^{* *}$ \\
3-AM (12 mg/kg) + LPS & $466 \pm 70$ & $52 \pm 7$ & $68 \pm 8$ \\
3-AM (24 mg/kg) + LPS & $550 \pm 80$ & $67 \pm 8$ & $73 \pm 9$ \\
3-CM (12 mg/kg) + LPS & $492 \pm 65$ & $57 \pm 6$ & $67 \pm 9$ \\
3-CM (24 mg/kg) + LPS & $619 \pm 47 *$ & $76 \pm 7 *$ & $89 \pm 7 *$ \\
DF (12 mg/kg) + LPS & $408 \pm 46$ & $49 \pm 7$ & $61 \pm 8$ \\
DF (24 mg/kg) + LPS & $385 \pm 65$ & $53 \pm 6$ & $64 \pm 7$ \\
\hline
\end{tabular}

${ }^{a} \mathrm{C} 57 \mathrm{BL} / 6 \mathrm{~J}$ mice received daily MPTP injections $(20 \mathrm{mg} / \mathrm{kg}$, s.c.) for 7 consecutive days. Each morphinan was administered $30 \mathrm{~min}$ before every injection of MPTP for the last 3 days. Animals were sacrificed $24 \mathrm{~h}$ after the last MPTP injection. Each value is the mean \pm sE from 12 animals. ${ }^{*} P<0.01$ vs. saline + saline; $* P<0.05, * * P<0.02$, and $* * * P<0.01$ vs. saline + MPTP, respectively.

nan analogs on DA neurons. To extend our previous in vitro finding that 3-HM protected DA neurons against LPS-induced neurotoxicity (4), a bilateral intrastriatal infusion of the LPS ( $2 \mu \mathrm{g}$ in a volume of $2 \mu \mathrm{l}$ of PBS) in vivo model was first used. LPS infusion caused a significant decrease in the number of TH-ir neurons (26\% of control) in SNpc of the injected group compared with the vehicle-injected controls (Fig. 2A). There was a corresponding reduction in the content of DA ( $43 \%$ of control) and its metabolites, DOPAC (37\% of control) and HVA (52\% of control), in LPS-injected striatum (Table 1). Daily injections with morphinans resulted in various degrees of protection on DA neurons in SNpc and recovery of DA levels in striatum (Fig. $2 A, B$ and Table 1). Of the five analogs tested, 3-HM was the most potent; it protected DA neurons by up to $75 \%$ that of the control (Fig. 2A). LPS-induced reduction in striatal DA levels was dose-dependently protected by 3-HM to $66 \%$ in a low dose and $90 \%$ in a high dose (Table 1).

The second in vivo model used involved daily injections of MPTP (20 mg/ $\mathrm{kg}$, s.c.), which induced a dramatic decrease of TH-ir neurons (27\% of control) in SNpc compared with vehicle-injected controls (Fig. $3 A)$. There was a corresponding decrease in the content of DA (38\% of control) and its metabolites, DOPAC (34\% of control) and HVA (50\% of control), in MPTP-injected striatum (Table 2). Similar to the results in the LPS model, daily injections with morphinans for 3 days resulted in different degrees of protection on DA neurons in SNpc and on DA levels in striatum (Fig. 3A, $B$ and Table 2). Among the different analogs investigated, 3-HM was also the most potent compound protecting against MPTP-induced loss of DA neurons of up to $76 \%$ of control (Fig $3 A$ ). MPTPinduced decrease in striatal DA levels was dose-dependently protected by $3-\mathrm{HM}$ to $54 \%$ in a low dose and
$78 \%$ in a high dose (Table 2). To determine whether morphinans affect the entry of MPTP to the brain, we had measured the brain MPP + concentrations at different time points after the last injection of MPTP in both control and 3-HM treated mice. The result showed no significant difference of MPP + levels in either group 1, 2, 3, 4, or $6 \mathrm{~h}$ after the last injection of MPTP (Fig. 3C).

Locomotor activity and its tracing pattern in LPSand MPTP-treated animals were measured. Injections of both toxins caused significant hypolocomotor activities (Fig. 2B, Fig. 3B), which were partially recovered with the injections of morphinans. Corresponding to the neuroprotective studies shown above, of the different analogs tested, 3-HM showed the most potent effect in recovering these hypolocomotor activities.

\section{3-HM protected against MPTP/MPP ${ }^{+}$-elicited DA neurodegeneration in vitro}

Because of its high potency, 3-HM was used as a prototype to further delineate the mechanism of neuroprotection by using rat primary midbrain mixed neuron-glia cultures. 3-HM $(1-5 \mu \mathrm{M})$ or vehicle was added to neuron-glia cultures $30 \mathrm{~min}$ prior to the application of MPTP $(1 \mu \mathrm{M}) / \mathrm{MPP}^{+}(0.5 \mu \mathrm{M})$. Seven days later the damage of DA neurons was determined by $\left[{ }^{3} \mathrm{H}\right] \mathrm{DA}$ uptake assay and morphological evaluation, including TH-ir neuron counts and dendrite measurements. As shown in Fig. 4A, MPTP/ $\mathrm{MPP}^{+}$reduced DA uptake capacity by 25 to $40 \%$, respectively, which was completely prevented by $1-5$ $\mu \mathrm{M}$ (94-129\% of control in MPTP treatment and $87-118 \%$ of control in $\mathrm{MPP}^{+}$treatment, respectively). 3-HM alone increased DA uptake capacity of up to $185-226 \%$ that of control. 

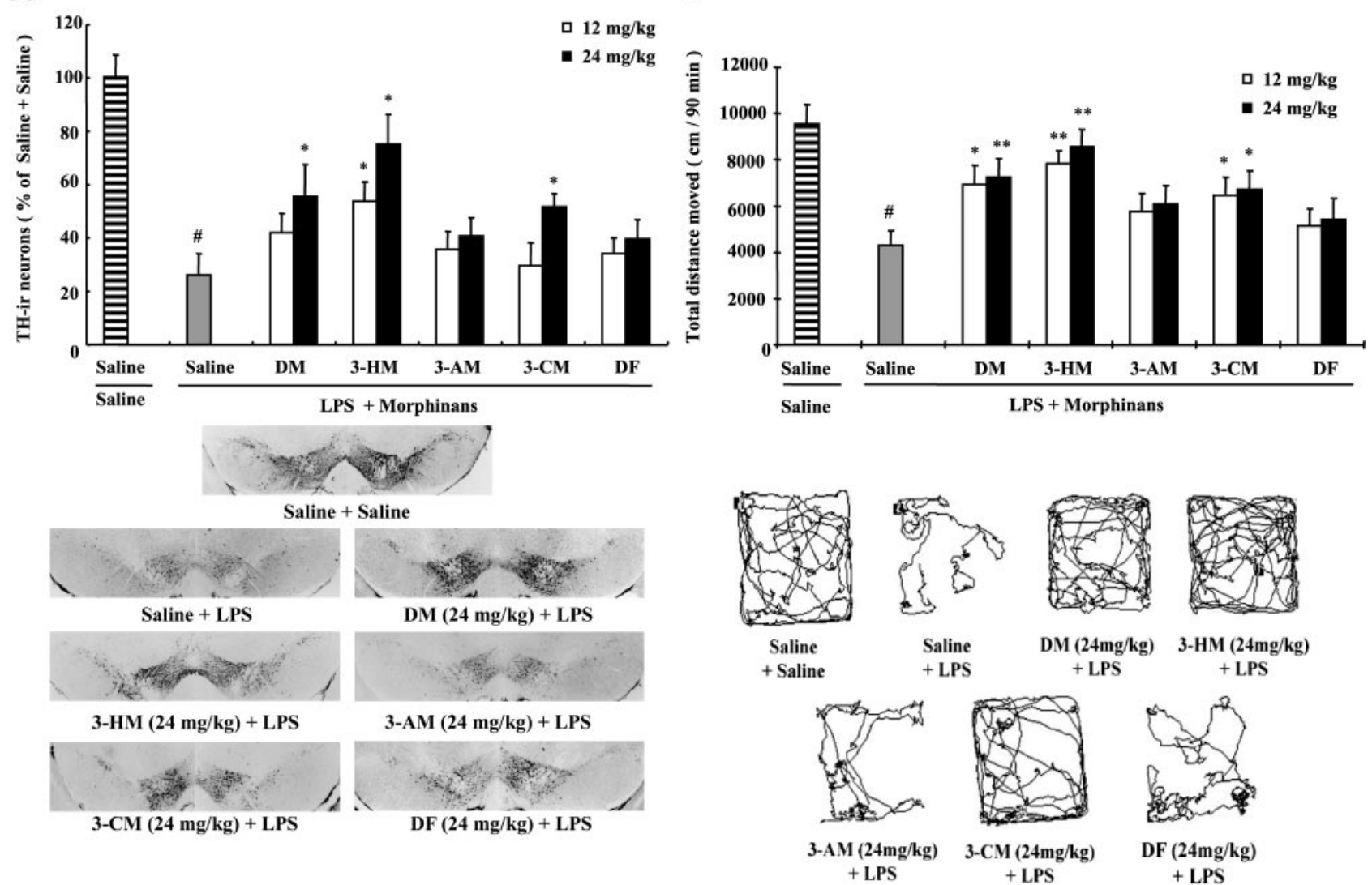

DM $(24 \mathrm{mg} / \mathrm{kg}) \quad 3-\mathrm{HM}(24 \mathrm{mg} / \mathrm{kg})$ + LPS $\quad+$ LPS

Figure 2. 3-HM was the most potent morphinan in attenuating the loss of DA neurons in SNpc and behavior damage induced by LPS in vivo. Morphinan compounds, including parent compound DM and its analogs 3-HM, 3-AM, 3-CM, and DF, were administered with a low and high dose (12 or $24 \mathrm{mg} / \mathrm{kg}$, i.p., respectively) $4 \mathrm{~h}$ and $30 \mathrm{~min}$ before intrastriatal LPS (2 $\mu \mathrm{g}$ in a volume of $2 \mu \mathrm{l}$ of PBS) injection to C57BL/6J mice. Mice were sacrificed $3 \mathrm{wk}$ after LPS injection. Total numbers of TH-ir neurons throughout $\mathrm{SNpc}$ were counted. Results are expressed as the mean \pm se from 6 animals $(A)$. ${ }^{\#} P<0.01$ vs. saline + saline; $* P<0.05$ vs. saline + LPS. Representative pictures $(24 \mathrm{mg} / \mathrm{kg}$ morphinans + LPS $)$ are shown $(40 \times)(A)$. In the behavioral study, results are expressed as the mean \pm se from 12 animals $(B) .{ }^{\#} P<0.01$ vs. saline + saline; ${ }^{*} P<0.05$ and $* * P<$ 0.01 vs. saline + LPS, respectively. Representative tracing patterns $(24 \mathrm{mg} / \mathrm{kg}$ morphinans + LPS $)$ are shown $(B)$.

Cell count analysis of DA neuronal numbers in the cultures revealed that MPTP $/ \mathrm{MPP}^{+}$induced loss of DA neurons by nearly $37 \%$ and $43 \%$, respectively, compared with vehicle-treated control cultures (Fig. 4B). $\mathrm{MPTP} / \mathrm{MPP}^{+}$-induced shortening of neurite length was parallel to that of neuronal number (Fig. 4C). Furthermore, the degree of loss of neuronal number and neurite length was comparable to that of DA uptake (Fig. 4A). Pretreatment with 3-HM (5 $\mu \mathrm{M})$ significantly attenuated $\mathrm{MPTP} / \mathrm{MPP}^{+}$-induced loss of DA neurons (127\% and $138 \%$ of control) and neurites $(135 \%$ and $145 \%$ of control) (Fig. $4 B$ ). The number of DA neuron and the length of neurites in the cultures treated with 3-HM ( $5 \mu \mathrm{M})$ alone were $216 \%$ and $233 \%$ vs. control, respectively.

Morphologically, in addition to the reduction in the abundance of DA neurons, the neurites of the remaining DA neurons in $\mathrm{MPTP} / \mathrm{MPP}^{+}$-treated cultures were significantly damaged compared with vehicle-treated control cultures, which were characterized by shorter, thinner, and broken neurites and, in the worst circumstance, even disappeared. In the
MPTP $/ \mathrm{MPP}^{+}$-treated cultures pretreated with 3-HM, DA neurons were significantly more numerous, and neurites were less damaged than in cultures treated with MPTP/MPP ${ }^{+}$alone (Fig 4C). Cultures treated with 3-HM alone had much more abundant neurites and healthier cell bodies compared with vehicletreated control cultures (Fig. 4C).

\section{Neuroprotective effect of 3-HM against MPP ${ }^{+}$. induced DA neurotoxicity was glia dependent}

We previously reported the neurotrophic activity of 3 -HM in rat primary midbrain mixed neuron-glia cultures (4), but the mechanism of how 3-HM exerted a neurotrophic effect was not understood. To determine whether the neurotrophic effect of 3-HM contributed to the neuroprotection on DA neurons from $\mathrm{MPP}^{+}$induced damage, the following experiments were performed. In neuron-enriched cultures, $\mathrm{MPP}^{+}(0.5 \mu \mathrm{M})$-induced direct DA neurotoxicity was revealed by a decrease in DA uptake (59\% of control), which was 
A
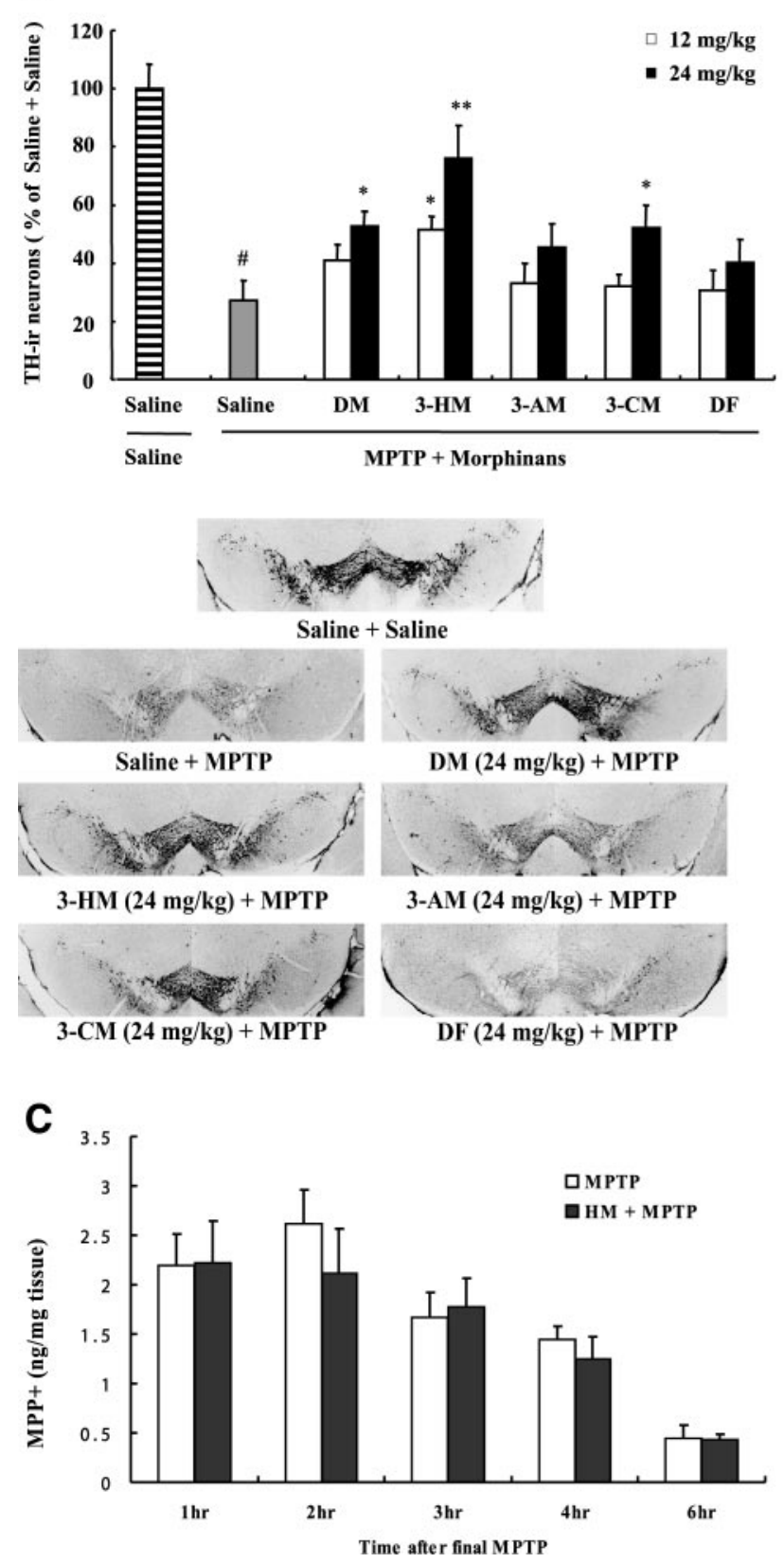

B
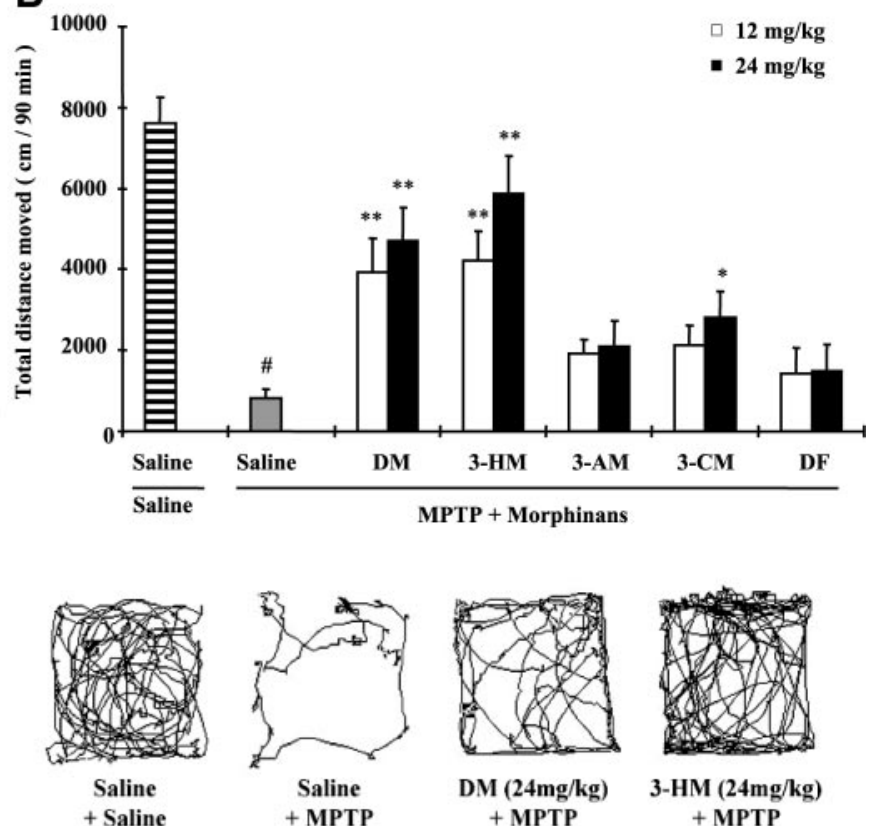

DM (24mg/kg) 3-HM (24mg/kg) + MPTP + MPTP

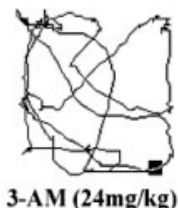

$3-\mathrm{AM}(24 \mathrm{mg} / \mathrm{kg}$ + MPTP
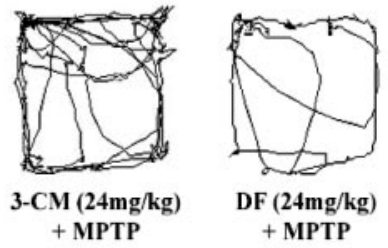

Figure 3. 3-HM was the most potent morphinan in attenuation of the loss of DA neurons in $\mathrm{SNpc}$ and behavior damage induced by MPTP in vivo. C57BL/6J mice received daily MPTP injection (20 mg/kg, s.c.) for 7 consecutive days. Each morphinan (12 or $24 \mathrm{mg} / \mathrm{kg}$, i.p.) was administered $30 \mathrm{~min}$ before every MPTP injection for the last 3 days. Animals were sacrificed $24 \mathrm{~h}$ after the last MPTP injection. Total numbers of TH-ir neurons throughout SNpc were counted $(A)$. Results are expressed as the mean \pm sE from 6 animals. $\# P<$ 0.01 vs. saline + saline; $* P<0.05$ and $* * P<0.01$ vs. saline + MPTP, respectively. Representative pictures $(24 \mathrm{mg} / \mathrm{kg}$ morphinans+MPTP) are shown $(40 \times)(A)$. In the behavioral study, results are expressed as the mean \pm SE from 12 animals $(B) . \# P<0.01$ vs. saline + saline; $* P<0.05$ and $* * P<0.01$ vs. saline + MPTP, respectively. Representative tracing patterns are shown $(24 \mathrm{mg} / \mathrm{kg}$ morphinans + MPTP) $(B) . \mathrm{MPP}+$ levels in mouse striatal extract after MPTP and 3-HM/MPTP treatment. 3-HM did not significantly affect changes in the MPP + level after the last MPTP administration. Results are expressed as the mean $\pm \mathrm{SE}$ from 7 animals $(C)$.

similar to that in neuron-glia cultures (50\% of control). However, 3-HM protected DA neurons in neuron-glia cultures (101\% of control) but not in neuron-enriched cultures $(71 \%$ of control), indicating that neuroprotection of 3-HM was mediated through glia cells. Based on the finding that 3-HM itself enhanced the capacity of DA uptake in neuron-glia cultures (210\% of control) but not in neuron-enriched cultures (113\% of control), we conclude there was a close correlation between the neurotrophic action and neuroprotection, and that glial cells were the major players in the neuroprotection afforded by 3-HM (Fig. 5).
Neurotrophic factors released from astroglia contributed to the neuroprotective effect of 3-HM against $\mathrm{MPP}^{+}$-induced DA neurotoxicity

In co-cultures of neuron-astroglia (adding $50 \%$ of astroglia back to neuron-enriched cultures) and neuronmicroglia (adding 10\% of microglia back to neuronenriched cultures), 3-HM (5 $\mu \mathrm{M})$ alone increased $\left[{ }^{3} \mathrm{H}\right] \mathrm{DA}$ uptake in the cultures with astroglia $(199 \%$ of control) but not microglia ( $103 \%$ of control), indicating that astroglia, not microglia, were the mediators of the neurotrophic action by 3-HM (Fig. 5). These results 
A

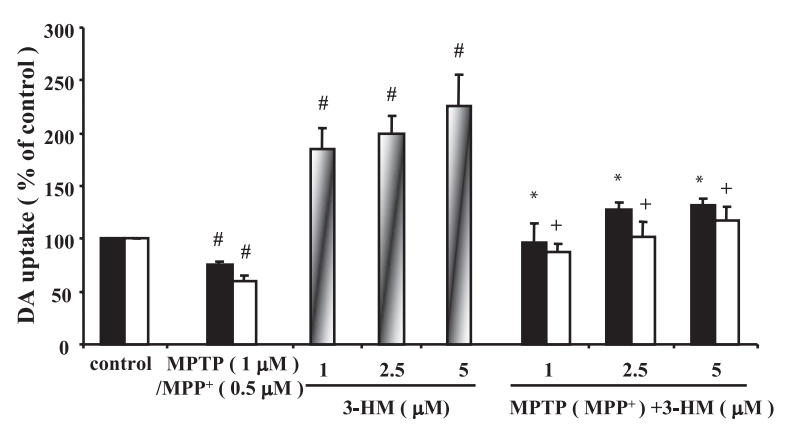

B

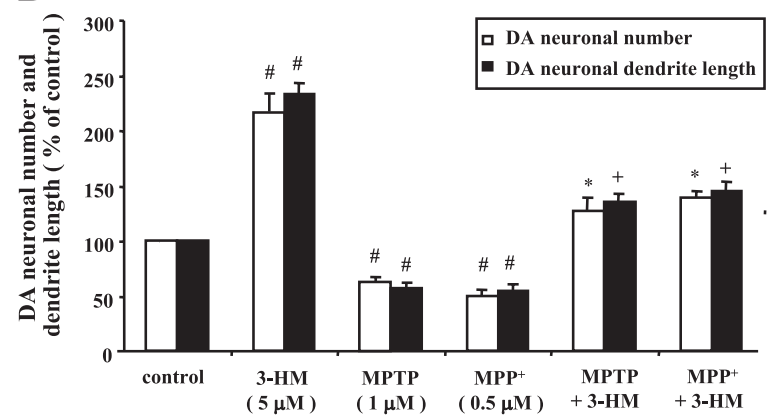

C
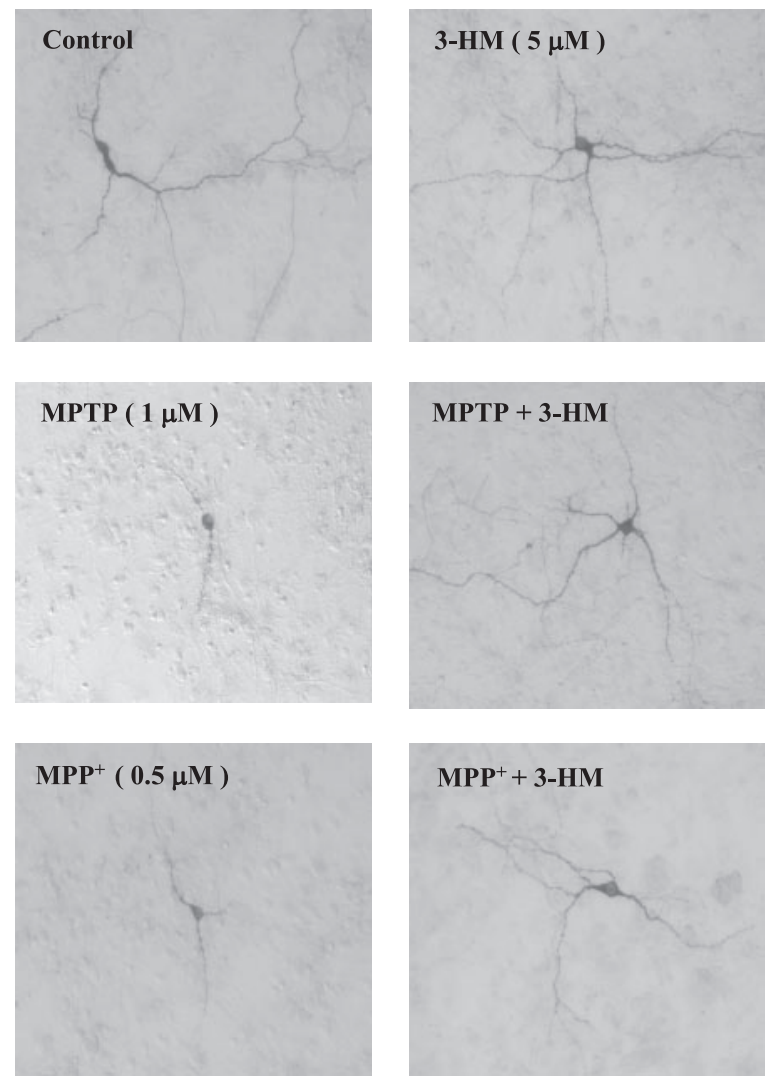

Figure 4. 3-HM protected against $\mathrm{MPTP} / \mathrm{MPP}^{+}$-elicited DA neurodegeneration in vitro. Rat primary midbrain mixed neuron-glia cultures seeded in a 24-well culture plate at $5 \times$ $10^{5} /$ well were pretreated with 3 -HM $(1-5 \mu \mathrm{M})$ for $30 \mathrm{~min}$ before the addition of $\operatorname{MPTP}(1 \mu \mathrm{M}) / \mathrm{MPP}^{+}(0.5 \mu \mathrm{M})$. Seven days later, MPTP $/ \mathrm{MPP}^{+}$-induced DA neurotoxicity was quantified by $\left[{ }^{3} \mathrm{H}\right]$ DA uptake assay $(A)$ and immunocytochemical analysis $(B)$, including counting the number of TH-ir neu-

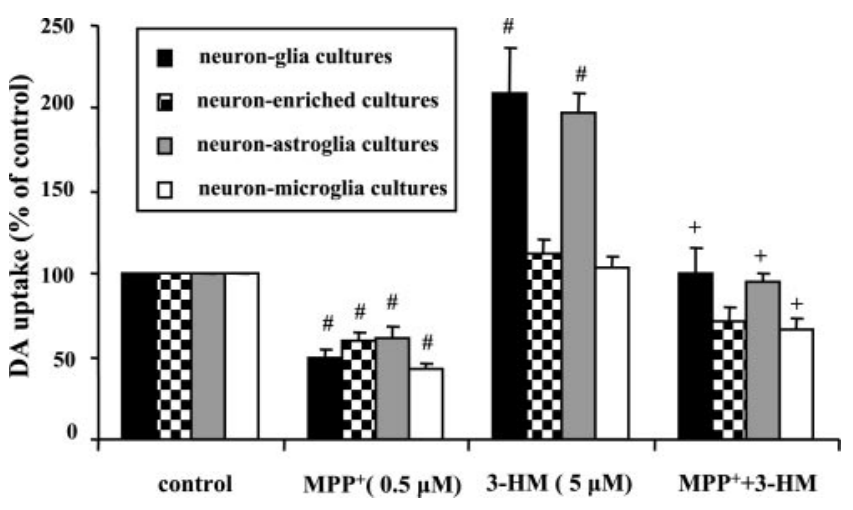

Figure 5. Astroglia is the source of neurotrophic and related neuroprotective effect of 3-HM against $\mathrm{MPP}^{+}$-induced DA neurodegeneration. Rat primary midbrain mixed neuron-glia cultures were seeded in a 24-well culture plate at $5 \times$ $10^{5}$ /well; primary neuron-enriched cultures were obtained by adding Ara-c to neuron-glia cultures at a final concentration of $7.5 \mu \mathrm{M}$ to suppress glial proliferation $24 \mathrm{~h}$ after seeding the cells; Primary neuron-astroglia cocultures were obtained by suppressing microglial proliferation with $1.5 \mathrm{mM} \mathrm{LME} 24 \mathrm{~h}$ after seeding the cells. Reconstituted neuron-microglia cultures were established by adding $10 \%\left(5 \times 10^{4} /\right.$ well $)$ of microglia back to neuron-enriched cultures. All above-mentioned cultures were pretreated with 3-HM $(5 \mu \mathrm{M})$ for 30 min before the addition of $\mathrm{MPP}^{+}(0.5 \mu \mathrm{M})$; DA neurotoxicity was quantified by $\left[{ }^{3} \mathrm{H}\right] \mathrm{DA}$ uptake assay $7-9$ days after treatment with $\mathrm{MPP}^{+}$. Results are expressed as a percentage of vehicletreated control cultures and are the mean \pm sE from 4-5 independent experiments in triplicate. ${ }^{\#} P<0.05$ vs. vehicletreated control cultures; ${ }^{+} P<0.05$ vs. $\mathrm{MPP}^{+}$-treated cultures.

were consistent with the findings that pronounced protection afforded by 3-HM against $\mathrm{MPP}^{+}$-induced DA neurotoxicity was observed in neuron-astroglia cocultures (Fig. 5).

Based on this information, we hypothesized that release of neurotrophic factors from astroglia by 3-HM was responsible for the robust neurotrophic effects. Further study using RT and real-time PCR revealed increases in the gene expressions of an array of neurotrophic factors, including endothelial growth factor (epidermal growth factor), neurotrophin 3 (NT3), glia cell line-derived neurotrophic factor (GDNF), astrogliaderived neurotrophic factor (ADNF), transforming growth factor $\beta 1$ (TGF- $\beta 1$ ) and transforming growth factor $\alpha$ (TGF- $\alpha)$, from astroglia treated with 3-HM (5 $\mu \mathrm{g} / \mathrm{ml}$ ) (Table 3). The peak time of gene expression at the mRNA level for most of the factors was between 3 and $6 \mathrm{~h}$ after 3 -HM administration. Among the factors examined, the expression of EGF appeared first, which peaked at $3 \mathrm{~h}$ ( $196 \%$ of control), followed by GDNF, NT3, TGF- $\beta 1$, and TGF- $\alpha$ (167\%, $164 \%, 151 \%$, and $144 \%$ of control, respectively); at $6 \mathrm{~h}$ after treatment,

rons and measuring the length of neurites. Representative pictures from 3 experiments were showed $(C)$. Results are expressed as a percentage of vehicle-treated control cultures and are the mean \pm SE from 3 independent experiments. ${ }^{\#} P<0.05$ vs. vehicle-treated control cultures; $* P<0.05$ and ${ }^{+} \mathrm{P}<0.05$ vs. MPTP $/ \mathrm{MPP}^{+}$-treated cultures, respectively. 


\begin{tabular}{|c|c|c|c|c|}
\hline \multirow[b]{2}{*}{ Neurotrophic factors } & \multicolumn{4}{|c|}{ Gene expression ( $\%$ of control) } \\
\hline & $3 \mathrm{~h}$ & $6 \mathrm{~h}$ & $12 \mathrm{~h}$ & $24 \mathrm{~h}$ \\
\hline EGF & $196 \pm 34^{* *}$ & $127 \pm 13$ & $123 \pm 15$ & $133 \pm 3$ \\
\hline GDNF & $167 \pm 13 * * *$ & $146 \pm 9 * *$ & $123 \pm 25$ & $139 \pm 10$ \\
\hline NT3 & $164 \pm 10^{* * *}$ & $133 \pm 8^{* *}$ & $103 \pm 14$ & $101 \pm 4$ \\
\hline TGF- $\beta 1$ & $151 \pm 13 * *$ & $149 \pm 9 * *$ & $131 \pm 11^{*}$ & $125 \pm 8$ \\
\hline TGF- $\alpha$ & $144 \pm 12 * *$ & $88 \pm 6$ & $115 \pm 14$ & $76 \pm 10$ \\
\hline $\mathrm{ADNF}$ & $124 \pm 13$ & $159 \pm 17 * *$ & $109 \pm 10$ & $125 \pm 9$ \\
\hline
\end{tabular}

${ }^{a}$ Primary mixed glial cells were prepared from the whole brains of 1-day-old Fisher 344 rat pups. Cells $\left(5 \times 10^{7}\right)$ were seeded in a $150 \mathrm{~cm}^{2}$ cultures flask. After a confluent monolaver of glia cells had been obtained, microglia were shaken off. After three consecutive passages, a purity of $>98 \%$ of astroglia was achieved and treated with 3-HM ( $5 \mu \mathrm{M})$ for 1, 3, 6, 12, and $24 \mathrm{~h}$. Astroglia were then detached with trypsin-EDTA and total RNA was isolated with Trizol reagent, followed by purification. RT and real-time PCR were utilized to detect gene expression of a series of neurotrophic factors using forward and reverse primers for the selected genes. Results are expressed as a percentage of vehicle-treated control cultures and are the mean $\pm \mathrm{sE}$ from 3 independent experiments in triplicate. ${ }^{*} P<0.05, * * P<0.01$, and $* * * P<0.005$ vs. vehicle-treated control cultures, respectively.

the factors with increased gene expression were ADNF, TGF- $\beta 1$, GDNF, and NT3 (159\%, 149\%, 146\%, and $133 \%$ of control, respectively); TGF- $\beta 1$ was the one that still maintained the level to some degree $(131 \%$ of control) $12 \mathrm{~h}$ after the treatment. The protein levels of most of these neurotrophic factors were below the detecting limit of the assay.

In another study, Western blot analysis (Fig. 6A, B) indicated that 3-HM $(5 \mu \mathrm{g} / \mathrm{ml})$ enhanced the expression of acetylated histone $\mathrm{H} 3$ in astroglia by up to 2.7-fold as soon as $1 \mathrm{~h}$ after treatment and maintained the level at 2.6-, 2.5-, 2-, and 2.5-fold of control after 3, 6,12 , and $24 \mathrm{~h}$ treatment, respectively, whereas valproic acid (VPA) served as a positive control (Chen, P. et al., unpublished results). The observation that the level of total histone $\mathrm{H} 3$ showed no change (Fig. $6 A, B$ ) indicated that the increased acetylated histone $\mathrm{H} 3$ was not due to the alteration of total histone H3.

\section{Prevention of MPTP $/ \mathrm{MPP}^{+}$-induced microgliosis by 3-HM was through inhibiting ROS production}

In neuron-glia cultures, the neuroprotection afforded by $3-\mathrm{HM}$ was increased by $51 \%$ increase in DA uptake (Fig. 5), which may be due mainly to the neurotrophic action from astroglia indicated by the $35 \%$ of increase in DA uptake in neuron-astroglia cocultures (Fig. 5). Although we did not see the neurotrophic effect in neuron-microglia cocultures because 3-HM alone did not increase the capacity of DA uptake, significant enhancement of DA uptake $(>20 \%)$ was still found in the cultures treated with $\mathrm{MPP}^{+}$and 3-HM, indicating that microglia was another target in 3-HM-provided neuroprotection against $\mathrm{MPP}^{+}$-elicited damage.

$\mathrm{MPTP} / \mathrm{MPP}^{+}$have been shown to induce a rapid reactive microgliosis after causing the direct DA neurodegeneration in mice $(20,21)$. Previous work from our laboratory indicated that $\mathrm{MPTP} / \mathrm{MPP}^{+}$-induced reactive microgliosis was closely associated with an increased production of oxygen free radicals $(20,22)$, including extracellular superoxide and iROS from microglia. Therefore, we determined whether 3-HM was able to reduce MPTP/MPP ${ }^{+}$-induced ROS generation.
Production of extracellular superoxide was determined in mouse primary midbrain mixed neuron-glia cultures pretreated for 30 min with 3-HM $(0.25-1 \mu \mathrm{M})$ prior to the addition of MPTP $(0.5 \mu \mathrm{M}) / \mathrm{MPP}^{+}(0.25$ $\mu \mathrm{M})$. Fresh 3-HM was added back to the cultures at days 2 and 5 after MPTP/MPP ${ }^{+}$treatment for replenishment. On days 4 and 7 , release of extracellular superoxide from activated microglia was measured using WST-1 as described in Materials and Methods. As shown in Fig. 7A, $\boldsymbol{B}$, treatment of cultures with 0.5 and $1 \mu \mathrm{M}$ 3-HM dose-dependently inhibited MPTP (0.5 $\mu \mathrm{M}) / \mathrm{MPP}^{+}(0.25 \mu \mathrm{M})$-induced superoxide production at the desired time points. 3-HM alone showed no effect on superoxide production.

Next, the effect of 3-HM on MPTP/ $/ \mathrm{MPP}^{+}$-induced iROS production was studied. Primary midbrain mixed neuron-glia cultures were treated in the same manner as mentioned above and iROS were determined at days 4 and 7 after MPTP $(0.5 \mu \mathrm{M}) / \mathrm{MPP}^{+}(0.25 \mu \mathrm{M})$ treatment. As shown in Fig. 8A, B, on day 4, 3-HM (0.25-0.5 $\mu \mathrm{M})$ dose-dependently inhibited the levels of iROS induced by MPTP $(0.5 \mu \mathrm{M})$. $\mathrm{MPP}^{+}(0.25 \mu \mathrm{M})$-induced iROS production was also decreased by $0.5-1 \mu \mathrm{M} 3-\mathrm{HM}$ on day 7 in a dose-dependent manner. Hence, these results demonstrated that 3-HM was capable of reducing MPTP $/ \mathrm{MPP}^{+}$-elicited reactive microgliosis-induced iROS production.

Taken together, these results demonstrated that 3-HM effectively inhibited MPTP/ $\mathrm{MPP}^{+}$-induced extracellular superoxide and iROS production, and suggested that the inhibition of reactive microgliosis through decreasing ROS productions was another important mechanism associated with the neuroprotective effect of 3-HM in addition to the neurotrophic action.

\section{3-HM did not produce psychotropic side effects}

Saline-treated animals exhibited basal locomotor activity and circling activity $($ Fig. $\mathbf{9 A}, \boldsymbol{B})$ as we demonstrated previously $(6,7)$. The repeated treatment of DM or its major metabolite, phencyclidine (PCP)-like dextrorphan (DX), significantly increased locomotor activity and circling behavior, although these behavioral re- 


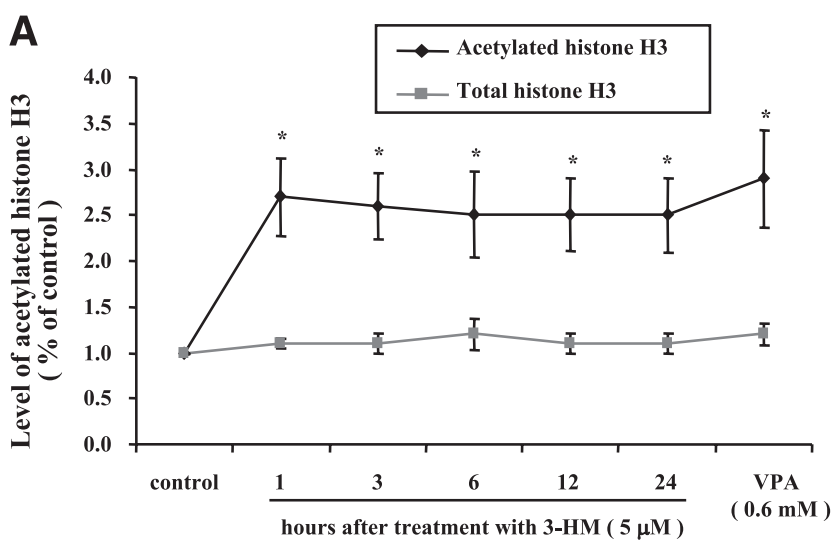

B

$\begin{array}{llllllll}3-\mathrm{HM}(5 \mu \mathrm{M}) & 0 & 1 & 3 & 6 & 12 & 24 & \text { VPA }(0.6 \mathrm{mM})\end{array}$
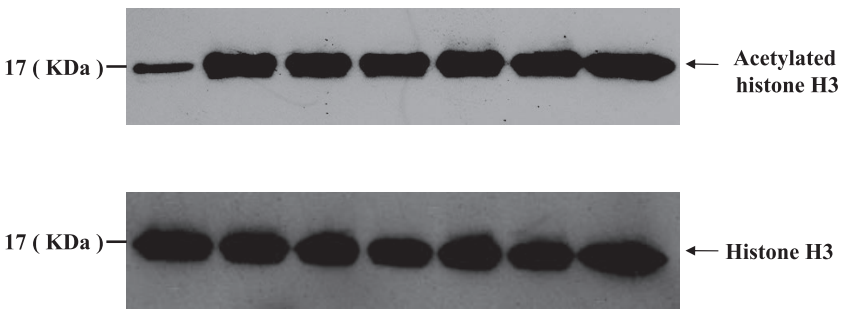

Figure 6. 3-HM up-regulated acetylated histone H3 in astroglia by Western blot analysis. Primary astroglia-enriched cultures treated with 3-HM $(5 \mu \mathrm{M})$ for $1,3,6,12$, and $24 \mathrm{~h}$ and VPA $(0.6 \mathrm{mM})$ for $24 \mathrm{~h}$, then cells were harvested for Western blot of acetylated and regular histone H3 protein. Autoradiographs were analyzed by densitometry. Quantified results are expressed as a percentage of vehicle-treated control cultures and are the mean \pm SE from 3 independent experiments $(A)$. $* P<0.05$ vs. vehicle-treated control cultures. Representative autographs are shown $(B)$.

sponses were less than PCP (Fig. 9A, B). 3-HM was the one that had the least behavioral responses mentioned above compared with DM and DX (Fig. 9A, B). For conditioned place preference (CPP), DM, DX, and PCP produced significant CPP whereas no CPP was observed in saline-treated animals. 3-HM did not show selective CPP effects that seen in DM- or DX-treated animals, which was comparable to other morphinans, such as 3-AM, 3-CM, and DF. Taken together, behavioral study indicated that 3-HM exerted negligible psychotropic effect compared with parent compound DM or its major metabolite DX.

\section{DISCUSSION}

In this study we report that of the five analogs of DM investigated, 3-HM provided the greatest degree of neuroprotection on DA neurons in the nigro-striatal pathway against LPS- and MPTP-elicited damage both in vivo and in vitro. The potent neuroprotective effect of 3-HM was attributed to its neurotrophic factor-releasing activity from astroglia and the inhibition of microgliosis. Note that 3-HM, a natural endogenous metabolite of DM, exerted a more potent neuroprotection than its parent compound.

In the in vivo study, 3-HM almost completely (up to $90 \%$ of the control in a high concentration of 24 $\mathrm{mg} / \mathrm{kg}$, i.p.) prevented DA neuronal loss in SNpc after MPTP or LPS treatment (Fig. $2 A, B$, Fig. $3 A, B$ ) and protected the DA depletion in striatum (Tables 1 and 2 ). The degree of neuroprotection by 3-HM shown in this study represents one of the most potent neuroprotective agents that have been reported (23-26). Most of the neuroprotective compounds are capable of protecting DA neurons in SNpc, but have much less efficacy in protecting DA content in striatum $(23,27-31)$. In this study, 3-HM showed equal potency in protecting DA neurons in SNpc and the DA content in striatum. The efficacy of neuroprotection was well correlated with the amelioration of behavioral damage, which revealed that 3-HM was the most potent one in attenuating both LPS-

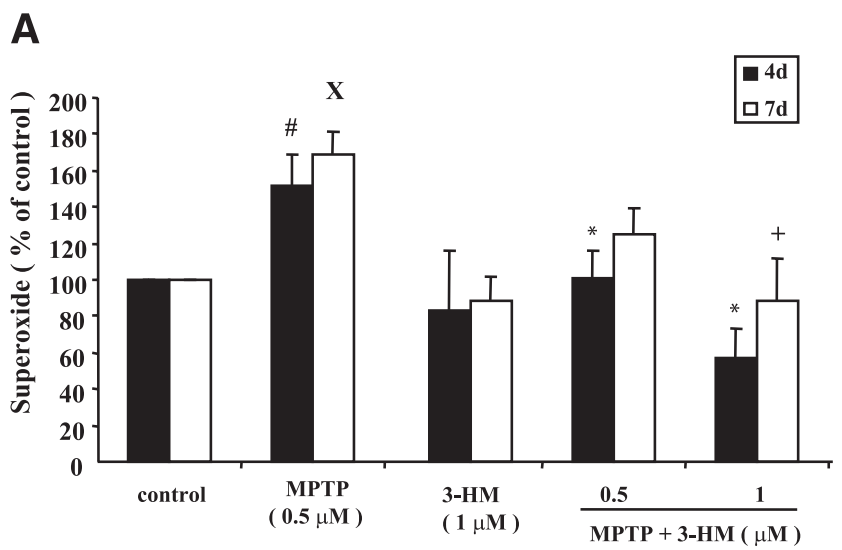

B

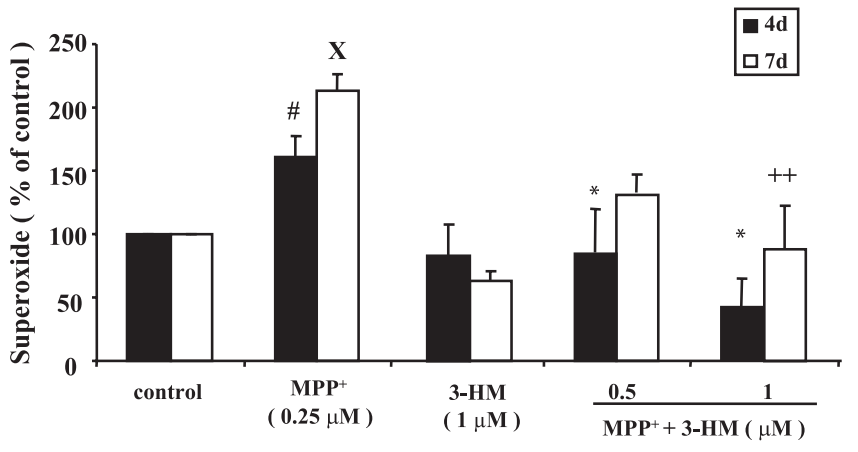

Figure 7. 3-HM prevented MPTP/ $\mathrm{MPP}^{+}$-induced microgliosis by inhibiting the production of extracellular superoxide. Primary C57BL/6J mice midbrain mixed neuron-glia cultures were seeded in 96-well plates at $1 \times 10^{5} /$ well. Cultures were pretreated for $30 \mathrm{~min}$ with $3-\mathrm{HM}(0.5-1 \mu \mathrm{M})$ prior to treatment with $0.5 \mu \mathrm{M}$ MPTP $(A)$ or $0.25 \mu \mathrm{M} \mathrm{MPP}^{+}(B)$. Two and 5 days after MPTP $/ \mathrm{MPP}^{+}$treatment, 3-HM $(0.5-1 \mu \mathrm{M})$ was added again to 3-HM-containing cultures, respectively. At days 4 and 7, the production of extracellular superoxide was determined. Results are expressed as a percentage of vehicletreated control cultures and are the mean $\pm \mathrm{SE}$ from 3 independent experiments performed in triplicate. ${ }^{\#} P<0.05$ and ${ }^{\mathrm{x}} P<0.05$ vs. vehicle-treated control cultures at days 4 and 7 , respectively; $* P<0.05,{ }^{+} P<0.05$, and ${ }^{++} P<0.01$ vs. $\mathrm{MPTP} / \mathrm{MPP}^{+}$-treated cultures on day 4 and 7 , respectively. 


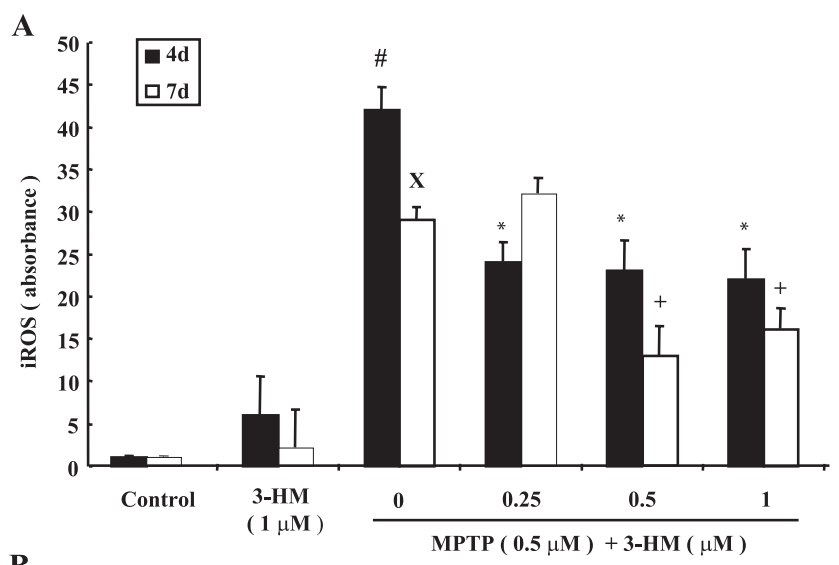

B

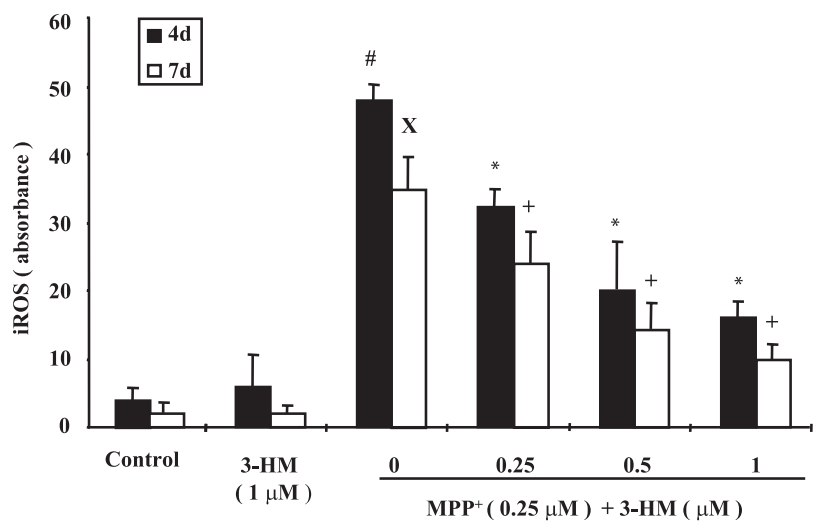

Figure 8. 3-HM prevented MPTP $/ \mathrm{MPP}^{+}$-induced microgliosis by inhibiting iROS production. Primary C57BL/6J mice midbrain mixed neuron-glia cultures were seeded in 96-well plates at $1 \times 10^{5} /$ well. Cultures were pretreated for $30 \mathrm{~min}$ with 3-HM $(0.25-1 \mu \mathrm{M})$ prior to treatment with $0.5 \mu \mathrm{M}$ MPTP $(A)$ or $0.25 \mu \mathrm{M} \mathrm{MPP}^{+}(B)$. Two and 5 days after MPTP $/ \mathrm{MPP}^{+}$ treatment, 3-HM $(0.25-1 \mu \mathrm{M})$ was added again to $3-\mathrm{HM}$ containing cultures. The production of iROS was determined on days 4 and 7 . Results are expressed as fluorescence absorbance units and are the mean \pm sE from 3 independent experiments performed at least three times. ${ }^{\#} P<0.05$ and ${ }^{x} P<0.05$ vs. vehicle-treated control cultures at days 4 and 7 , respectively; $* P<0.05$ and ${ }^{+} P<0.05$ vs. $\mathrm{MPTP} / \mathrm{MPP}^{+}$treated cultures at days 4 and 7 , respectively.

and MPTP-induced hypolocomotor activity. In addition, we have demonstrated that relatively high doses of 3-HM exerted negligible psychotropic side effects compared with DM and its major metabolite, DX, which is an important consideration for the potential for longterm clinical usage in PD patients. Furthermore, both MPTP and intrastriatal LPS injection models first target dopaminegic terminal field, which in turn cause retrograde neurodegeneration of dopaminergic neurons in the substantia nigra. Our data showing that 3-HM prevents both the degeneration of nigral dopaminergic neurons and the depletion of dopamine level in the striatum suggest a possibility that targeting dopaminergic terminal field can be considered as a strategy for the preventive therapy of PD.

Mechanistic studies using primary midbrain neuronglia cocultures revealed two glia-dependent mechanisms for the potent neuroprotection afforded by 3-HM in an MPTP model.
Astroglia mediated the 3-HM-induced neurotrophic effect

In the in vitro culture systems containing astroglia, such as neuron-glia and neuron-astroglia cultures, 3-HM alone-treated groups showed a significant neurotrophic effect revealed by the dramatically enhanced DA uptake after 3-HM-alone treatment compared with vehicle-treated control cultures (Fig. 5), which was closely correlated with the increased number of DA neurons and the prolonged length of DA neurites in the 3-HM alone-treated group (Fig. 4B). However, parent compound DM itself did not show neurotrophic effect as indicated by increased DA uptake, DA neuron numbers, and neurites $(2,3)$. Thus, the neurotrophic effect of 3-HM is critical for explaining the more potent neuroprotective action of 3-HM on DA neurons in the $\mathrm{SNpc}$ and DA content in the striatum.

Further study indicated that the astroglia-mediated neurotrophic effect after 3-HM treatment was via increasing the gene expression of an array of neurotrophic factors in astroglia such as EGF, NT3, GDNF, ADNF, TGF- $\alpha$, and TGF- $\beta 1$. The magnitudes of the increase in the expression of these neurotrophic factors at the mRNA level were modest; probably the neurotrophic effect of 3-HM could not be attributed to a single factor, since the concentration of any individual factor released might not have been great enough to be effective. Thus, it was likely that the synergy among the released factors may account for the neurotrophic effect of 3-HM. The synergistic neurotrophic effect from the combined trophic factors has been documented (32-35).

To search for the possible mechanism for the neurotrophic action of 3-HM, we studied the acetylation of histone based on the recent findings in our lab about VPA, a drug widely used to treat seizures (36) and manic/depressive phases of bipolar mood disorder (36, 37). Histone deacetylase (HDAC) is an enzyme that catalyzes the removal of acetyl group from lysine residues of histones $(38,39)$. VPA was reported to directly inhibit HDAC at therapeutic levels, causing histone hyperacetylation $(38,39)$. HDAC has been strongly implicated in the modulation of gene expression as well as life span in a variety of organisms (40). In addition, VPA has been shown to activate the cell survival factor presumably through inhibition of $\operatorname{HDAC}(36,41)$. Our lab recently found that VPA exerted neuroprotection on DA neurons and released neurotrophic factors from astroglia, which was associated with the increase in acetylation of chromatin histone $\mathrm{H} 3$ through the inhibition of HDAC (Chen, P. et al., unpublished results). The ability of VPA to covalently modify histone structures through enhancing acetylation can trigger changes in the gene expression of distinct downstream factors, such as neurotrophic factors (Chen, P. et al., unpublished results).

Western blot analysis from this study revealed that 3-HM $(5 \mu \mathrm{M})$ increased the acetylation of histone $\mathrm{H} 3$ in astroglia cultures whereas total histone $\mathrm{H} 3$ showed no 
Figure 9. 3-HM had negligible psychotropic effects. C57BL/6J mice received a daily i.p. injection of each morphinan [20 or $40 \mathrm{mg} / \mathrm{kg}$, dissolved in saline $(2 \mathrm{ml} / \mathrm{kg})]$ for 7 days. "Total distance moved in $\mathrm{cm}$ " by the animals in the horizontal locomotor activity was measured for $30 \mathrm{~min}$ after the last treatment $(A)$. After measuring locomotor activity, the "absolute turn angular" was analyzed in a 3 min monitoring period using an automated video tracking system in order to examine circling behavior (marginal activity) $(B)$. For a conditioned place preference (CPP) test, mice received an i.p. injection of saline just before entering the white or black compartment. Each compound was administered immediately before the mice were placed in the white compartment (see Materials and Methods). Scores were calculated from the differences in the time spent in the white compartment in the testing and pretesting phases $(C)$. Results are expressed as the mean $\pm \mathrm{SE}$ from 10 animals $(A, B)$ or 15 animals $(C)$. $* P<0.05$, ** $P<0.01$ and $* * * P<$ 0.001 vs. saline; ${ }^{\mathrm{x}} P<0.05$ vs. PCP $2.5 \mathrm{mg} / \mathrm{kg}$; ${ }^{\#} P<0.05$ vs. corresponding dose of $\mathrm{DM} ;{ }^{+} \mathrm{P}<$ 0.05 , and ${ }^{++} P<0.01$ vs. corresponding dose of DX.
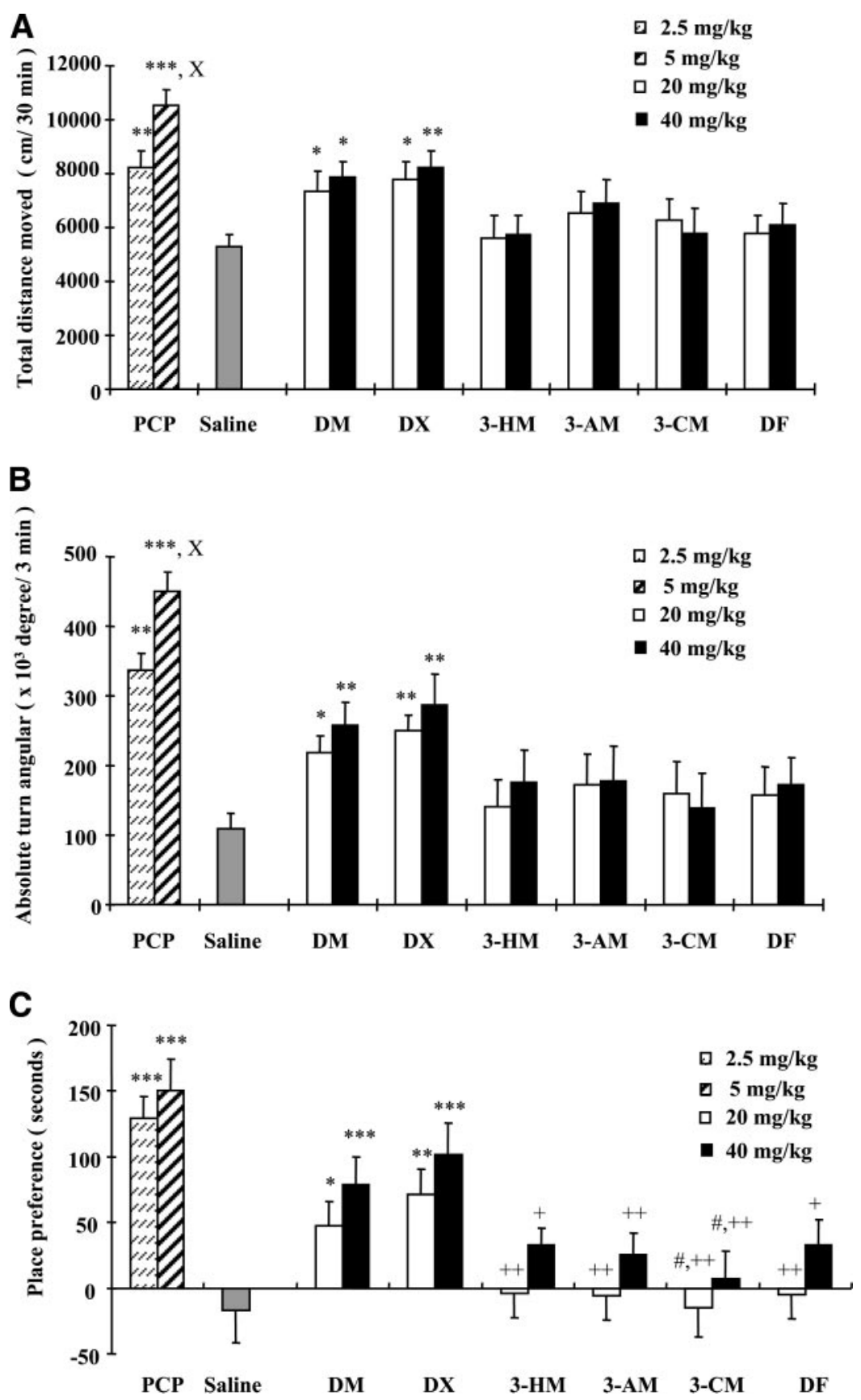

increased level. This finding suggested that 3-HM increased gene expression of neurotrophic factors through the increase in acetylation of chromatin histone. Studies are under way to further determine whether 3-HM-induced the increase in the acetylation of histone H3 is due to the inhibition of HDAC or the increase in the activity of histone acetylase.

\section{Microglia participated in 3-HM-mediated neuroprotection against MPTP-induced DA neuronal damage}

We previously reported that the neuroprotective effect of DM and 3-HM in primary midbrain neuronglia cultures treated with LPS was associated with the inhibition of LPS-induced microglia activation $(2,4)$, which in turn slowed down the inflammatory process and protected DA neurons from LPS-induced damage. In this study, the anti-inflammatory effect of 3-HM in the MPTP model was mediated through a mechanism different from the above-mentioned LPS model. The anti-inflammatory effect of 3-HM in the MPTP model was due to the reduced reactive microgliosis elicited after MPTP treatment as evidenced by the decrease in the production of ROS from microglia, including extracellular superoxide and iROS. Reactive microgliosis after MPTP treatment has been documented by our laboratory and others $(20,22,42$, 43). It is believed that the initial damage or death of DA neurons caused by MPTP could signal the activa- 
tion of microglia through either the release of soluble factors from damaged neurons or the loss of cell inhibition between neurons and microglia (22, 44$47)$. Reactive microgliosis may release more proinflammatory and neurotoxic factors to augment the neuroinflammatory process and cause further damage on the remaining DA neurons. Thus, the continuing processes among the death of DA neurons, the secondary activation of microglia (microgliosis), and further neuronal damage would create a vicious "self-propelling" cycle and trigger a progressive neurodegeneration $(48-50)$.

In vitro results from this study were consistent with the above-mentioned hypothesis of reactive microgliosis. Figures 7 and 8 show a delayed increase in the production of superoxide and iROS from microglia 4 days after MPTP treatment in primary midbrain mixed neuron-glia cultures, and these increases were significantly reduced by pretreatment with 3-HM. Previous reports from our laboratory indicated that microglial NADPH oxidase mediated the production of superoxide and iROS after MPTP treatment $(20,42)$, and both $\mathrm{DM}$ and 3-HM were capable of inhibiting the activation of this ROS-generating enzyme $(2,4,22)$. Thus, it was highly likely that the anti-inflammatory effect of 3-HM in the MPTP model resulted from the inhibition of MPTP-induced production of ROS from microglia after reactive microgliosis.

In addition to the mechanistic studies, our findings also provide significant advances in the concept of drug development for PD therapy. Current DA replacement therapy fails to slow down the progression of PD. Our findings presented in this study provide critical insights into novel avenues aimed at halting the disease progression. The unique features of our findings are described below.

\section{Astroglia is a source of neurotrophic factors for the neuroprotection of 3-HM}

It is well documented that neurotrophic factors are vital for neuronal survival (51-61). Several clinical trials aimed at rescuing DA neurons by either direct infusion of GDNF or gene delivery to the brain of PD patients were conducted in several pharmaceutical companies in the past few years, but few of these trials succeeded. Our findings show that astroglia can be an important target for the source of neurotrophic factors and provide a new strategy for the release of various astroglial neurotrophic factors, which has proved to be extremely effective in protecting the survival of DA neurons in $\mathrm{SNpc}$ and the DA levels in striatum in both in vitro and in vivo rodent PD models. This approach has great advantages over the methods of direct delivery of a single neurotrophic factor to the brain due to its potent neuroprotection, noninvasive nature, negligible side effects, and low cost.
Inhibition of microglial over-activation is a potential target for the neuroprotection of 3-HM

It is generally accepted that neuroinflammation generated by the activation of microglia plays a critical role in neurodegenerative disorders, such as PD. We and others have documented that over-activation of microglia produces an array of proinflammatory and neurotoxic factors such as free radicals, cytokines, chemokines, and prostaglandins, which may damage the surrounding neurons and cause neurodegeneration $(11,13,21,62-66)$. Among the various factors released from microglia, superoxide generated by NADPH oxidase plays the most critical role in the subsequent neuroinflammatory process and neurodegeneration by exerting direct damage on DA neurons and promoting the production of other proinflammatory factors such as NO, TNF $\alpha$, IL- 1 , and $\mathrm{PGE}_{2}(4,11$, $13,63)$. Thus, NADPH oxidase is an ideal target for developing anti-inflammatory drugs due to 1 ) its dual roles played in inflammation-related neuronal damage, and 2) a high degree of cell type specificity because NADPH oxidase is highly expressed in immune cells such as microglia, macrophages, or neutrophils, but is in low abundance in T or B cells and is not found in most of the other cell types $(63,66)$. Our research also shows that this anti-inflammatory approach is equally effective in preventing the over-activation of microglia caused by either a direct activation induced by LPS or an indirect reactive microgliosis elicited by MPTP/MPP ${ }^{+}$.

In summary, we show the potent neuroprotective effect of 3-HM against LPS- and MPTP-induced DA neuronal damage in in vivo and in vitro PD models through dual functions of increasing the gene expression of a series of neurotrophic factors from astroglia and decreasing superoxide and iROS production from microglia caused by the reactive microgliosis. In view of significant attenuation of behavioral damage induced by both LPS and MPTP and the negligible psychotropic side effect of 3-HM, plus the fact that 3-HM, being a small molecule, can be taken orally, 3-HM may serve as a prototype drug for the development of neuroprotective compounds that comprise both neurotrophic and anti-inflammatory properties to slow down or even halt the progression of $\mathrm{PD}$.

This research was supported by the Intramural Research Program of the NIEHS/NIH; by a grant (M103KV01001306K220201310) from the Brain Research Center from 21st Century Frontier Research Program funded by the Ministry of Science and Technology, Republic of Korea; by a grant of the Korea Health 21 R\&D Project (A020007), Ministry of Health and Welfare; by Korea FDA; and by BK 21 Project, Korea Research Foundation. Matching fund (by Green Cross Corp., Korea) in response to a grant (M103KV01001306K220201310) was used for this study. Equipment at the Institute of Pharmaceutical Sciences Kangwon National University) was used for this study. We appreciate the technical support given by Drs. Jie Liu, Jong-Seok Chae, Belinda Wilson, Kooyeon Lee, Po See Chen, Zheng-Yi Lee, S. F Ali, Myung Eun Jung, Sung-Jen Wei, Xuefei Wu, and Li Qian. Thanks to Drs. Jef French and Jie Liu for reviewing and providing comments in the preparation of this manuscript. 


\section{REFERENCES}

1. Olanow, C. W., and Tatton, W. G. (1999) Etiology and pathogenesis of Parkinson's disease. Annu. Rev. Neurosci. 22, 123-144

2. Liu, Y., Qin, L., Li, G., Zhang, W., An, L., Liu, B., and Hong, J. S. (2003) Dextromethorphan protects dopaminergic neurons against inflammation-mediated degeneration through inhibition of microglial activation. J. Pharmacol. Exp. Ther. 305, 212218

3. Zhang, W., Wang, T., Qin, L., Gao, H. M., Wilson, B., Ali, S. F., Zhang, W., Hong, J. S., and Liu, B. (2004) Neuroprotective effect of dextromethorphan in the MPTP Parkinson's disease model: role of NADPH oxidase. FASEB J. 18, 589-591

4. Zhang, W., Qin, L., Wang, T., Wei, S. J., Gao, H. M., Liu, J., Wilson, B., Liu, B., Zhang, W., Kim, H. C., and Hong, J. S. (2005) 3-hydroxymorphinan is neurotrophic to dopaminergic neurons and is also neuroprotective against LPS-induced neurotoxicity. FASEB J. 19, 395-397

5. Kim, H. C., Nabeshima, T., Jhoo, W. K., Ko, K. H., Kim, W. K., Shin, E. J., Cho, M., and Lee, P. H. (2001) Anticonvulsant effects of new morphinan derivatives. Bioorg. Med. Chem. Lett. 11, $1651-1654$

6. Kim, H. C., Shin, C. Y., Seo, D. O., Jhoo, J. H., Jhoo, W. K., Kim, W. K., Shin, E. J., Lee, Y. H., Lee, P. H., and Ko, K. H. (2003) New morphinan derivatives with negligible psychotropic effects attenuate convulsions induced by maximal electroshock in mice. Life Sci. 72, 1883-1895

7. Shin, E. J., Nah, S. Y., Kim, W. K., Ko, K. H., Jhoo, W. K., Lim, Y. K., Cha, J. Y., Chen, C. F., and Kim, H. C. (2005) The dextromethorphan analog dimemorfan attenuates kainate-induced seizures via sigmal receptor activation: comparison with the effects of dextromethorphan. Br. J. Pharmacol. 144, 908-918

8. Hows, M. E. P., Ashmeade, T. E., Billinton, A., Perren, M. J., Austin. A. A., Virley, D. J., Organ, A. J., and Shah, A. J. (2004) High-performance liquid hromatography/tandem mass spectrometry assay for the determination of 1-methyl-4-phenyl pyridinium $(\mathrm{MPP}+)$ in brain tissue homogenates. J. Neurosci. Methods 137, 221-226

9. Kim, H.C, Jhoo, W.K, Shin, E. J., and Bing, G. (2000) Selenium deficiency potentiates methamphetamine-induced nigral neuronal loss; comparison with MPTP model. Brain Res. 862, 247-252

10. Ali, S. F., Newport, G. D., Holson, R. R., Slikker, W., Jr., and Bowyer, J. F. (1994) Low environmental temperatures or pharmacologic agents that produce hypothermia decrease methamphetamine neurotoxicity in mice. Brain Res. 658, 33-38

11. Wang, T., Pei, Z., Zhang, W., Liu, B., Langenbach, R., Lee, C., Wilson, B., Reece, J. M., Miller, D. S., and Hong, J. S. (2005) $\mathrm{MPP}+$-induced COX-2 activation and subsequent dopaminergic neurodegeneration. FASEB J. 19, 1134-1136

12. Liu, B., Du, L., and Hong, J. S. (2000) Naloxone protects rat dopaminergic neurons against inflammatory damage through inhibition of microglia activation and superoxide generation. J. Pharmacol. Exp. Ther. 293, 607-617

13. Zhang, W., Wang, T., Pei, Z., Miller, D. S., Wu, X., Block, M. L., Wilson, B., Zhang, W., Zhou, Y., Hong, J. S., and Zhang, J. (2005) Aggregated alpha-synuclein activates microglia: a process leading to disease progression in Parkinson's disease. FASEB J. 19, 533-542

14. Liu, J., He, Y. Y., Chignell, C. F., Clark, J., Myers, P., Saavedra, J. E., and Waalkes, M. P. (2005) Limited protective role of V-PYRRO/NO against cholestasis produced by alpha-naphthylisothiocyanate in mice. Biochem. Pharmacol. 70, 144-151

15. Tan, A. S., and Berridge, M. V. (2000) Superoxide produced by activated neutrophils efficiently reduces the tetrazolium salt, WST-1 to produce a soluble formazan: a simple colorimetric assay for measuring respiratory burst activation and for screening anti-inflammatory agents. J. Immunol. Methods 238, 59-68

16. Peskin, A. V., and Winterbourn, C. C. (2000) A microtiter plate assay for superoxide dismutase using a water-soluble tetrazolium salt (WST-1). Clin. Chim. Acta 293, 157-166

17. Liu, Y., Qin, L., Wilson, B. C., An, L., Hong, J. S., and Liu, B. (2002) Inhibition by naloxone stereoisomers of beta-amyloid peptide (1-42) -induced superoxide production in microglia and degeneration of cortical and mesencephalic neurons. J. Pharmacol. Exp. Ther. 302, 1212-1219
18. Rosenkranz, A. R., Schmaldienst, S., Stuhlmeier, K. M., Chen, W., Knapp, W., and Zlabinger, G. J. (1992) A microplate assay for the detection of oxidative products using $2^{\prime}, 7^{\prime}$-dichlorofluorescin-diacetate. J. Immunol. Methods 156, 39-45

19. Wang, T., Liu, B., Zhang, W., Wilson, B., and Hong, J. S. (2004) Andrographolide reduces inflammation-mediated dopaminergic neurodegeneration in mesencephalic neuron-glia cultures by inhibiting microglial activation. J. Pharmacol. Exp. Ther. 308, 975-983

20. Gao, H. M., Liu, B., Zhang, W., and Hong, J. S. (2003) Critical role of microglial NADPH oxidase-derived free radicals in the in vitro MPTP model of Parkinson's disease. FASEB J. 17, 19541956

21. Wu, D. C., Jackson-Lewis, V., Vila, M., Tieu, K., Teismann, P., Vadseth, C., Choi, D. K., Ischiropoulos, H., and Przedborski, S. (2002) Blockade of microglial activation is neuroprotective in the 1-methyl-4-phenyl-1,2,3,6-tetrahydropyridine mouse model of Parkinson disease. J. Neurosci. 22, 1763-1771

22. Zhang, W., Wang, T., Qin, L., Gao, H. M., Wilson, B., Ali, S. F., Hong, J. S., and Liu, B. (2004) Neuroprotective effect of dextromethorphan in the MPTP Parkinson's disease model: role of NADPH oxidase. FASEB J. 18, 589-559

23. Eberhardt, O., Coelln, R. V., Kugleret, S., Lindenau, J., RathkeHartlieb, S., Gerhardt, E., Haid, S., Isenmann, S., Gravel, C., Srinivasan, A., et al. 2000) Protection by synergistic effects of adenovirus-mediated X-chromosome-linked inhibitor of apoptosis and glial cell line-derived neurotrophic factor gene transfer in the 1-methyl-4-phenyl-1,2,3,6-tetrahydropyridine model of Parkinson's disease. J. Neurosci. 20, 9126-9134

24. Cleren, C., Calingasan, N. Y., Chen, J., and Beal, M. F. (2005) Celastrol protects against MPTP- and 3-nitropropionic acidinduced neurotoxicity. J. Neurochem. 94, 995-1004

25. Zhou, H. F., Liu, X. Y., Niu, D. B., Li, F. Q., He, Q. H., and Wang, X. M. (2005) Triptolide protects dopaminergic neurons from inflammation-mediated damage induced by lipopolysaccharide intranigral injection. Neurobiol. Dis. 18, 441-449

26. Li, F. Q., Cheng, X. X., Liang, X. B., Wang, X. H., Xue, B., He, Q. H., Wang, X. M., and Han, J. S. (2003) Neurotrophic and neuroprotective effects of tripchlorolide, an extract of Chinese herb Tripterygium wilfordii Hook F, on dopaminergic neurons. Exp. Neurol. 179, 28-37

27. Yasuhara, T., Shingo, T., Muraoka, K., Kameda, M., Agari, T., Wen Ji, Y., Hayase, H., Hamada, H., Borlongan, C. V., and Date, I. (2005) Neurorescue effects of VEGF on a rat model of Parkinson's disease. Brain Res. 1053, 10-18

28. Peng, J., Stevenson, F. F., Doctrow, S. R., and Andersen, J. K. (2005) Superoxide dismutase/catalase mimetics are neuroprotective against selective paraquat-mediated dopaminergic neuron death in the substantial nigra: implications for Parkinson disease. J. Biol. Chem. 280, 29194-29198

29. Inden, M., Kondo, J., Kitamura, Y., Takata, K., Nishimura, K. Taniguchi, T., Sawada, H., and Shimohama, S. (2005) Proteasome inhibitors protect against degeneration of nigral dopaminergic neurons in hemiparkinsonian rats. J. Pharmacol. Sci. 97, 203-211

30. Datla, K. P., Bennett, R. D., Zbarsky, V., Ke, B., Liang, Y. F., Higa, T., Bahorun, T., Aruoma, O. I., and Dexter, D. T. (2004) The antioxidant drink effective microorganism-X (EM-X) pre-treatment attenuates the loss of nigrostriatal dopaminergic neurons in 6-hydroxydopamine-lesion rat model of Parkinson's disease. J. Pharm. Pharmacol. 56, 649-654

31. Cutillas, B., Ambrosio, S., and Unzeta, M. (2002) Neuroprotective effect of the monoamine oxidase inhibitor PF 9601N [N-(2-propynyl)-2-(5-benzyloxy-indolyl) methylamine] on rat nigral neurons after 6-hydroxydopamine-striatal lesion. Neurosci. Lett. 329, 165-168

32. Roussa, E., Farkas, L. M., and Krieglstein, K. (2004) TGF-beta promotes survival on mesencephalic dopaminergic neurons in cooperation with Shh and FGF-8. Neurobiol. Dis. 16, 300-310

33. Wood, T. K., McDermott, K. W., and Sullivan, A. M. (2005) Differential effects of growth/differentiation factor 5 and glial cell line-derived neurotrophic factor on dopaminergic neurons and astroglia in cultures of embryonic rat midbrain. J. Neurosci. Res. 80, 759-766

34. Erickson, J. T., Brosenitsch, T. A., and Katz, D. M. (2001) Brain-derived neurotrophic factor and glial cell line-derived neurotrophic factor are required simultaneously for survival of 
dopaminergic primary sensory neurons in vivo. J. Neurosci. 21, $581-589$

35. Krieglstein, K., Strelau, J., Schober, A., Sullivan, A., and Unsicker, K. (2002) TGF-beta and the regulation of neuron survival and death. J. Physiol. (Paris) 96, 25-30

36. Jeong, M. R., Hashimoto, R., Senatorov, V. V., Fujimaki, K., Ren, M., Lee, M. S., and Chuang, D. M. (2003) Valproic acid, a mood stabilizer and anticonvulsant, protects rat cerebral cortical neurons from spontaneous cell death: a role of histone deacetylase inhibition. FEBS Lett. 542, 74-78

37. Tunnicliff, G. (1999) Actions of sodium valproate on the central nervous system. J. Physiol. Pharmacol. 50, 347-365

38. Gottlicher, M., Minucci, S., Zhu, P., Kramer, O. H., Schimpf, A., Giavara, S., Sleeman, J. P., Lo Coco, F., Nervi, C., Pelicci, P. G., and Heinzel, T. (2001) Valproic acid defines a novel class of HDAC inhibitors inducing differentiation of transformed cells. EMBO J. 20, 6969-6978

39. Phiel, C. J., Zhang, F., Huang, E. Y., Guenther, M. G., Lazar, M. A., and Klein, P. S. (2001) Histone deacetylase is a direct target of valproic acid, a potent anticonvulsant, mood stabilizer, and teratogen. J. Biol. Chem. 276, 36734-36741

40. Chang, K. T., and Min, K. T. (2002) Regulation of lifespan by histone deacetylase. Ageing Res. Rev. 1, 313-326

41. De Sarno, P., Li, X., and Jope, R. S. (2002) Regulation of Akt and glycogen synthase kinase-3 beta phosphorylation by sodium valproate and lithium. Neuropharmacology 43, 1158-1164

42. Gao, H. M., Liu, B., Zhang, W., and Hong, J. S. (2003) Synergistic dopaminergic neurotoxicity of MPTP and inflammogen lipopolysaccharide: relevance to the etiology of Parkinson's disease. FASEB J. 17, 1957-1959

43. McGeer, P. L., Schwab, C., Parent, A., and Doudet, D. (2003) Presence of reactive microglia in monkey substantia nigra years after 1-methyl-4-phenyl-1,2,3,6-tetrahydropyridine administration. Ann. Neurol. 54, 599-604

44. Feng, Z. H., Wang, T. G., Li, D. D., Fung, P., Wilson, B. C., Liu, B., Ali, S. F., Langenbach, R., and Hong, J. S. (2002) Cyclooxygenase-2-deficient mice are resistant to 1-methyl-4-phenyl1, 2, 3, 6-tetrahydropyridine-induced damage of dopaminergic neurons in the substantia nigra. Neurosci. Lett. 329, 354-358

45. Wu, D. C., Teismann, P., Tieu, K., Vila, M., Jackson-Lewis, V., Ischiropoulos, H., and Przedborski, S. (2003) NADPH oxidase mediates oxidative stress in the 1-methyl-4-phenyl-1,2,3,6-tetrahydropyridine model of Parkinson's disease. Proc. Natl. Acad. Sci. U. S. A. 100, 6145-6150

46. Teismann, P., Vila, M., Choi, D. K., Tieu, K., Wu, D. C., Jackson-Lewis, V., and Przedborski, S. (2003) COX-2 and neurodegeneration in Parkinson's disease. Ann. N. Y. Acad. Sci. 991, 272-277

47. Sriram, K., Matheson, J. M., Benkovic, S. A., Miller, D. B., Luster, M. I., and O'Callaghan, J. P. (2002) Mice deficient in TNF receptors are protected against dopaminergic neurotoxicity: implications for Parkinson's disease. FASEB J. 16, 1474-1476

48. Liu, B., Gao, H. M., and Hong, J. S. (2003) Parkinson's disease and exposure to infectious agents and pesticides and the occurrence of brain injuries: role of neuroinflammation. Environ. Health Perspect. 111, 1065-1073

49. Block, M. L., and Hong, J. S. (2005) Microglia and inflammation-mediated neurodegeneration: multiple triggers with a common mechanism. Prog. Neurobiol. 76, 77-98

50. Liu, B., and Hong, J. S. (2003) Role of microglia in inflammation-mediated neurodegenerative diseases: mechanisms and strategies for therapeutic intervention. J. Pharmacol. Exp. Ther. $304,1-7$
51. Blum, M. (1998) A null mutation in TGF-alpha leads to a reduction in midbrain dopaminergic neurons in the substantia nigra. Nat. Neurosci. 1, 374-377

52. Jaumotte, J. D., and Zigmond, M. J. (2005) Dopaminergic innervation of forebrain by ventral mesencephalon in organotypic slice co-cultures: effects of GDNF. Brain Res. Mol. 134, $139-146$

53. Roussa, E., and Krieglstein, K. (2004) GDNF promotes neuronal differentiation and dopaminergic development of mouse mesencephalic neurospheres. Neurosci. Lett. 361, 52-55

54. Sun, Z. H., Lai, Y. L., Li, P., Zuo, H. C., and Xie, Z. P. (2004) GDNF augments survival and differentiation of TH-positive neurons in neural progenitor cells. Cell Biol. Int. 28, 323-325

55. Chen, Y., Ai, Y., Slevin, J. R., Maley, B. E., and Gash, D. M. (2005) Progenitor proliferation in the adult hippocampus and substantia nigra induced by glial cell line-derived neurotrophic factor Exp. Neurol. 196, 87-95

56. Kobori, N., Waymire, J. C., Haycock, J. W., Clifton, G. L., and Dash, P. K. (2004) Enhancement of tyrosine hydroxylase phosphorylation and activity by glial cell line-derived neurotrophic factor. J. Biol. Chem. 279, 2182-2191

57. Brizard, M., Carcenac, C., Bemelmans, A. P., Feuerstein, C., Mallet, J., and Savasta, M. (2005) Functional reinnervation from remaining DA terminals induced by GDNF lentivirus in a rat model of early Parkinson's disease. Neurobiol. Dis.

58. Muller, H. W., Junghans, U., and Kappler, J. (1995) Astroglial neurotrophic and neurite-promoting factors. Pharmacol. Ther. 65, 1-18

59. Canudas, A. M., Pezzi, S., Canals, J. M., Pallas, M., and Alberch, J. (2005) Endogenous brain-derived neurotrophic factor protects dopaminergic nigral neurons against transneuronal degeneration induced by striatal excitotoxic injury. Brain Res. Mol. 134, 147-154

60. Urbanics, R. (2001) NT-3. Takeda/Regeneron/Amgen. IDrugs 4, 820-824

61. Farkas, L. M., Dunker, N., Roussa, E., Unsicker, K., and Krieglstein, K. (2003) Transforming growth factor-beta(s) are essential for the development of midbrain dopaminergic neurons in vitro and in vivo. J. Neurosci. 23, 5178-5186

62. Gao, H. M., Jiang, J., Wilson, B., Zhang, W., Hong, J. S., and Liu, B. (2002) Microglial activation-mediated delayed and progressive degeneration of rat nigral dopaminergic neurons: relevance to Parkinson's disease. J. Neurochem. 81, 1285-1297

63. Qin, L., Liu, Y., Wang, T., Wei, S. J., Block, M. L., Wilson, B., Liu, B., and Hong, J. S. (2004) NADPH oxidase mediates lipopolysaccharide-induced neurotoxicity and proinflammatory gene expression in activated microglia. J. Biol. Chem. 279, 1415-1421

64. Qin, L., Liu, Y., Cooper, C., Liu, B., Wilson, B., and Hong, J. S (2002) Microglia enhance beta-amyloid peptide-induced toxicity in cortical and mesencephalic neurons by producing reactive oxygen species. J. Neurochem. 83, 973-983

65. Basu, A., Krady, J. K., O’Malley, M., Styren, S. D., DeKosky, S. T., and Levison, S. W. (2002) The type 1 interleukin-1 receptor is essential for the efficient activation of microglia and the induction of multiple proinflammatory mediators in response to brain injury. J. Neurosci. 22, 6071-6082

66. Gao, H. M., Hong, J. S., Zhang, W., and Liu, B. (2002) Distinct role for microglia in rotenone-induced degeneration of dopaminergic neurons. J. Neurosci. 22, 782-790

Received for publication March 2, 2006. Accepted for publication July 17, 2006. 